\title{
A Study of Multicriteria Decision Making for Supplier Selection in Automotive Industry
}

\author{
Nadia Jamil, Rosli Besar, and H. K. Sim \\ Faculty of Engineering and Technology, Multimedia University, Jalan Ayer Keroh Lama, 75450 Bukit Beruang, Melaka, Malaysia \\ Correspondence should be addressed to Nadia Jamil; diajh288@yahoo.com.my
}

Received 8 November 2012; Accepted 29 January 2013

Academic Editor: C. K. Kwong

Copyright ( 2013 Nadia Jamil et al. This is an open access article distributed under the Creative Commons Attribution License, which permits unrestricted use, distribution, and reproduction in any medium, provided the original work is properly cited.

\begin{abstract}
This paper is designed to present the effectiveness of group multicriteria decision making in automotive manufacturing company focusing on the selection of suppliers in Malaysia. The process of selecting suppliers is one of the most critical and challenging endeavor in any supply chain management. There are five decision making tools being analyzed in this study, namely, analytical hierarchy process (AHP), fuzzy analytical hierarchy process (FAHP), technique for order performance by similarity to ideal solution (TOPSIS), fuzzy technique for order performance by similarity to ideal solution (FTOPSIS), and fuzzy analytical hierarchy process integrated with fuzzy technique for order performance by similarity to ideal solution (FAHPiFTOPSIS). The scores of ranking among the suppliers in each MCDM tools (AHP, FAHP, TOPSIS, FTOPSIS, and FAHPiFTOPSIS) show significantly comparable variation. Scores of the best supplier is then compared to the lowest supplier for all MCDM tools whereby this reflects that the highest percentage goes to TOPSIS with scoring of 79.37\%. On the contrary, FAHPiFTOPSIS demonstrated the lowest score variation of $22.42 \%$ which indicates that FAHPiFTOPSIS is able to eliminate biasness in supplier selection process.
\end{abstract}

\section{Introduction}

A supply chain is a system which connects several departments from procurement of raw materials, to manufacturing, warehousing, and distribution of the products to the customers. Part of the contribution to supply chain complexity is the geographical outsourcing for cheaper supply and new market penetration. The complexity of supply chain is aggravated further when industry rely too much on multirange products and frequent introduction of new products as a strategy to meet different segmented market demands.

In automotive industry, such situation is rampant. The frequent introduction of new models and shorter product lifecycles compounded by fast order-delivery require high level of agility and flexibility of the suppliers, thereby, exacerbating the supply chain complexity. Hence, the right selection of supplier becomes more complicated. With the mounting complexity of supply chain, the selection of the suppliers becomes very challenging. The recent incident in Fukushima, Japan, devastated by massive earthquake and nuclear disaster, and major floods in Thailand, had affected severely many Malaysian industries as well as industries in other parts of the world [1]. Those Malaysian companies which have their suppliers associated with these companies have suffered critical production problems, in particular, automotive industry. Thus, purchasing function for each organization is increasingly seen as the most important role in supplier selection [2].

In the previous years the automotive industry has witnessed an unprecedented turmoil. Such crises had affected the European and Asian automotive industry and had gravely stricken the American automobile industry. The first half of 2009 had indeed been a very faltering year due to economy recession. Based on press report released by MAA [3] dated 19 January 2011 (Figure 1), sales of passenger cars in Malaysia had dropped by $7.5 \%$ from year-to-date September 2008 to September 2009 (see Table 1) affected by the bad economic slowdown.

The economy is currently in transition from recession to recovery in tandem with financial markets improvement. 
TABLE 1: Vehicles Sales in 2008 and 2009.

\begin{tabular}{|c|c|c|c|c|c|c|}
\hline \multirow{2}{*}{ Segment } & \multicolumn{3}{|c|}{ September } & \multicolumn{3}{|c|}{ Year-to-date-September } \\
\hline & 2009 & 2008 & Variance & 2009 & 2008 & Variance \\
\hline PV (passenger vehicles) & 42,039 & 46,476 & 0.90 & 361,463 & 392,393 & 0.92 \\
\hline CV (commercial vehicles) & 4,030 & 4,253 & 0.95 & 36,156 & 37,520 & 0.96 \\
\hline Total & 46,069 & 50,729 & 0.91 & 397,619 & 429,913 & 0.92 \\
\hline
\end{tabular}

TABLE 2: Summary of passenger and commercial vehicles produced and assembled in Malaysia from 1980 to 2010.

\begin{tabular}{lcccc}
\hline Year & Passenger cars & Commercial vehicles & $4 \times 4$ vehicles & Total vehicles \\
\hline 1980 & 80,422 & 23,805 & - & 104,227 \\
1985 & 69,769 & 37,261 & - & 11,873 \\
1990 & 116,526 & 63,181 & 11,253 & 107,030 \\
1995 & 231,280 & 45,805 & 27,235 & 288,338 \\
2000 & 295,318 & 36,642 & 45,623 & 359,195 \\
2005 & 422,225 & 95,662 & 28,551 & 563,510 \\
2006 & 377,952 & 96,545 & - & 503,048 \\
2007 & 403,245 & 38,433 & - & 441,678 \\
2008 & 484,512 & 46,298 & - & 530,810 \\
2009 & 447,002 & 42,267 & - & 489,269 \\
2010 & 522,568 & 45,147 & 567,715 \\
\hline
\end{tabular}

(Source: [3]).

Note:

(i) Passenger vehicle industry reclassified in January 2007 and includes all passenger carrying vehicles, that is, passenger cars, 4WD/SUV, window van, and MPV models.

(ii) Commercial vehicles also reclassified on 1 January 2007 and include trucks, prime movers, pick-up, panel vans, buses and others.

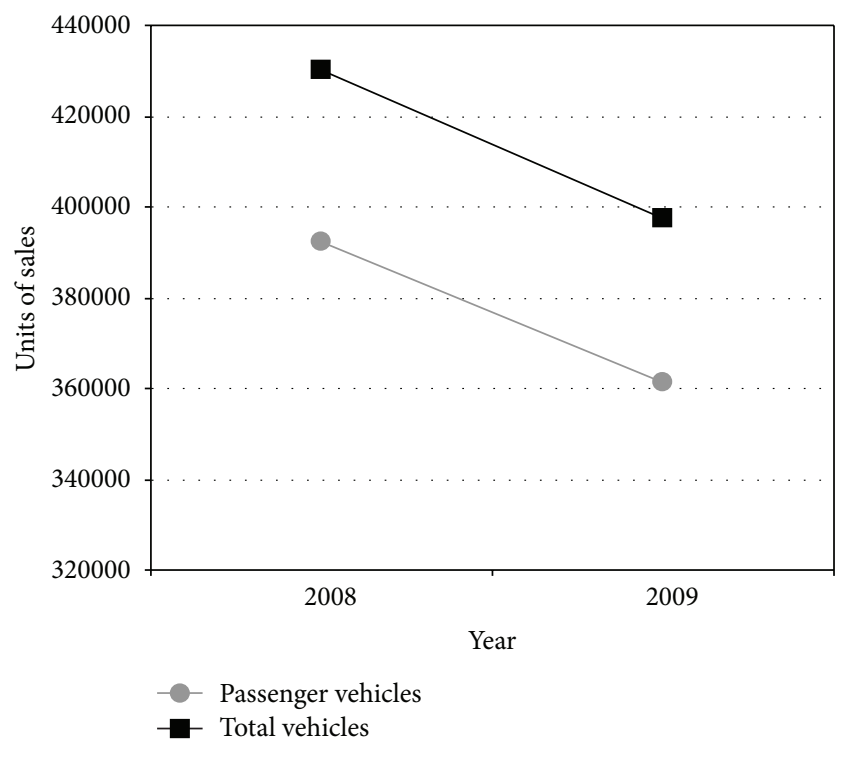

FIGURE 1: Vehicles sales in 2008 and 2009.

Year 2010 shows an increase trend in automotive industry. In 2010, the total industry volume of vehicles has picked up and increased to 605,156 units and, in fact, surpassed the highest recorded volume of 552,316 units in 2005.

Table 1 shows total vehicles produced and assembled from 1980 to 2010. From Table 2, total vehicles produced and assembled in 2010 were 605,156 units, increased by
$12.7 \%$ from 536,905 units produced the year before. In 1980 the production statistics shows only two types of vehicles: passenger cars and commercial vehicles. In 1990, the statistics had included production figures for 4WD vehicles. In 2007, there was a reclassification where passenger cars, $4 \mathrm{WD} / \mathrm{SUV}$, window van, and MPV models are all categorized as passenger vehicles. Commercial vehicles had been reclassified to include trucks, prime movers, pick-up, panel vans, bus, and others. The data in Table 1 is summarized graphically in Figure 2 which illustrates the bar graph for passenger and commercial vehicles produced and assembled in Malaysia from year 1980 to 2010 .

The data in Table 2 is summarized in bar graph shown in Figure 3 to highlight the significant increase in the number of passengers cars produced and assembled from 1980 to 2010. The total number of vehicles produced and assembled also showed a good progress of increment from year to year. To see the production trend of commercial vehicles from 2005 to 2010, Figure 3 is presented. From Figure 3, it is noted that the production of commercial vehicles did not exceed more than 100,000 units.

In Figure 3, the line graph representing the commercial vehicles illustrates a slight increment of production from 2009 to 2010 which is an increase of $6.8 \%$ of 45,147 units. In terms of percentage contribution of commercial vehicles to the market, it gradually increases from $8.8 \%$ in 2005 to $10.2 \%$ in 2010 [4].

In May 2011, based on a press release by Malaysian Automotive Association (MAA), automotive industry has 
TABLE 3: Summary of passenger and commercial vehicles produced and assembled in Malaysia from May 2010 to May 2011.

\begin{tabular}{lcccccc}
\hline \multirow{2}{*}{ Segment } & May & & \multicolumn{3}{c}{ Year-to-date May } \\
& 2011 & 2010 & Variance & 2011 & 2010 & 222,977 \\
\hline PV (passenger vehicles) & 40,936 & 46,259 & 0.88 & 228,816 & 1.03 \\
CV (commercial vehicles) & 5,109 & 4,624 & 1.1 & 26,597 & 24,133 \\
\hline Total & 46,045 & 50,883 & 0.9 & 255,413 & 247,110 \\
\hline
\end{tabular}

(Source: [3]).

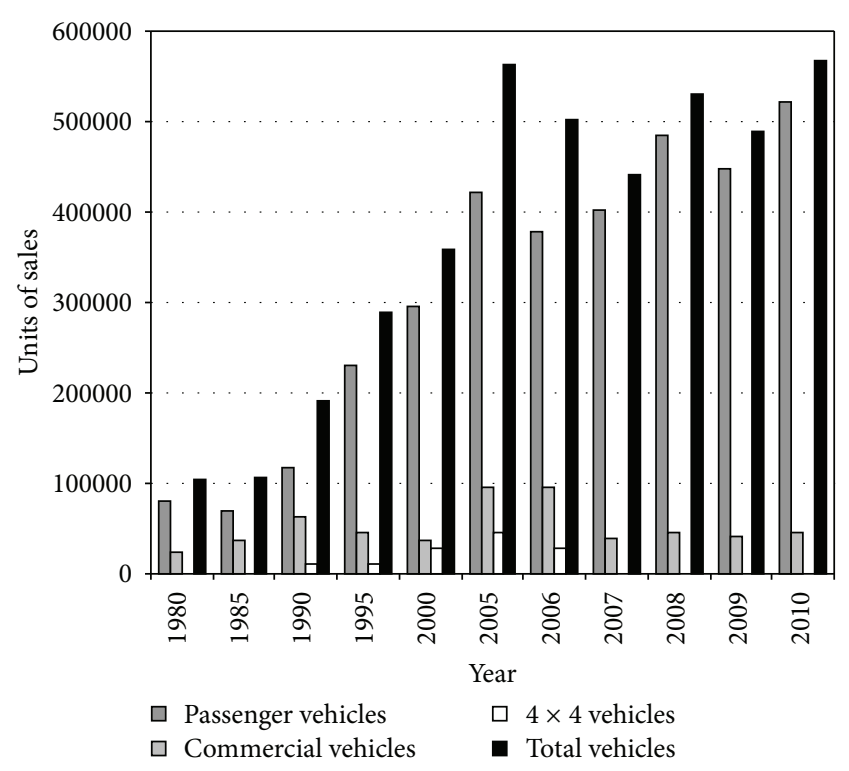

FIGURE 2: Bar graph of passenger and commercial vehicles produced and assembled in Malaysia from 1980 to 2010 [3].

shown a healthy increase of sales compared to the same period the year before (see Table 3). However, in comparison to sales in May 2010 and May 2011, it showed that there was a reduction of $11.5 \%$. This impact is due to the earthquake and tsunami which happened in Japan. More than $80 \%$ of the vehicles produced were dominated by passenger vehicles.

Currently, automotive industry is slowly and gradually shifting towards Asian countries, mainly due to high cost and saturation of automobile industry in the west and the increase in demand in Asia. The principal driving markets for Asian automobile industry are China, India, and ASEAN nations. The future of automotive industry in the Asian countries in particular Thailand, Philippines, Malaysia, and Indonesia is bright and promising because of the (ASEAN Free Trade Area) AFTA with tariffs currently at 0 to 5\% [5].

Malaysia is a country that has a long history of making cars. There are many local and international cars assemblers and manufacturers in the country. The employees of the industry are widely regarded as skilled, well educated, and trainable. Located strategically in the ASEAN region which has a population of more than 500 million people, Malaysia offers vast opportunities for global automotive and component manufacturers and suppliers to set up their manufacturing and distribution operations in the country. Mercedes-Benz assembly plant has proven its great prospect

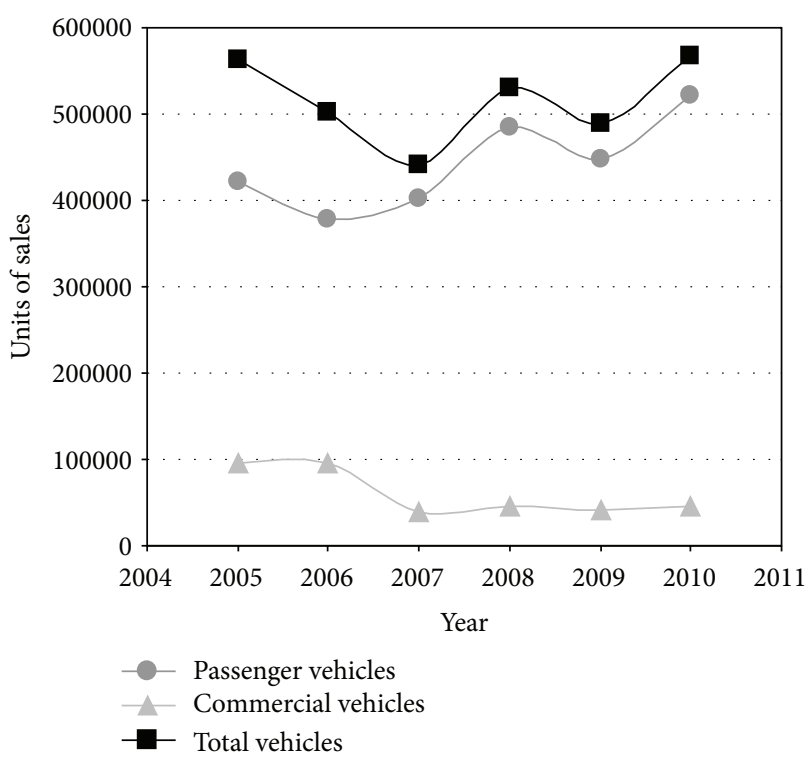

FIGURE 3: Line graph of passenger and commercial vehicles produced and assembled in Malaysia from 2005 to 2010 [3].

where its plant located in Pekan, Pahang, initially assembled only 4 units per day for one model and today assembles the S-Class, E-Class, and C-Class Mercedes with annual volume reaching 5,000 units [6].

Supplier selection decision process considers qualitative and quantitative criteria [2, 7]. It is a complex process whereby it involves many criteria, not necessarily quality, cost, and delivery. Such decision making process that involves multiple criteria is classified as a multicriteria decision making $(\mathrm{MCDM})$ process.

One of the MCDM is the analytical hierarchy process (AHP) whereby it is a theory of mathematical for decision making and measurement introduced by Saaty [8]. It assists decision makers to make effective decision based on its goals, criteria, and alternatives. AHP can be applied in making decisions which are unstructured and complex and consists of multiple criteria [9]. This method has been used in various areas including performance evaluation, supplier selection, credit scoring, project management, resource allocation, distribution channel management, inventory management, promotion and recruitment decisions, portfolio management, energy resources planning, technology management, financial planning, budgeting decisions, socioeconomic planning, common vote prediction, and conflict resolution [10]. The Technique for Order Preference by Similarity to the 
Ideal Solution (TOPSIS) is another mathematical model for MCDM. TOPSIS advocates two artificial alternatives, that is, the ideal alternative, the one which has the best level for all attributes considered, and the negative ideal alternative, the one which has the worst attribute values. TOPSIS selects the alternative that is the closest to the ideal solution and farthest from negative ideal alternative [11]. For the purpose of this research and without any prejudice to the others, two MCDM mathematical models have been selected, that is, AHP and TOPSIS.

In making decision for supplier selection, it is agreed by $[2,12]$ that the decisions made are very often involved by several decision makers. The purpose of having more people in decision making is to avoid any weaknesses or prejudice in the selection process. In order to make a decision which reflects human thinking, a system needs to be realistic. In making decision, decision makers prefer to evaluate a criterion with a certain level of tolerance rather than a fixed value judgment [13]. Due to this, one system which implies a human-like thinking model is introduced which is known as fuzzy logic [14].

This system describes a matter with a certain degree of characteristic which is also known as membership function. Membership function is a graphical representation which associates with the magnitude of input and ultimately determines an output response. There are different membership functions associated with each input and output response. Details about membership function are explained in Section 2.

The remainder of this paper is organized as follows: in Section 2, fuzzy concepts and the integration between fuzzy AHP and TOPSIS are explained; Section 3 shows the methodology used to conduct this study; Section 4 explained the results and discussion, finally in Section 5, the conclusions are presented.

\section{Background of Fuzzy Concepts}

Fuzzy sets can be simply defined as a set with fuzzy boundaries whereby the values of boundaries is multivalued unlike the two-valued Boolean logic. In the fuzzy theory, fuzzy set $X$ of universe $Y$ is defined by function $\mu_{X}(y)$ which is called the membership functions of set $X$. This notation can be expressed as follows [15]:

$$
\mu_{X}(y): Y \longrightarrow[0,1]
$$

where

$$
\begin{array}{cc}
\mu_{X}(y)=1 \quad \text { if } y \text { is totally in } X, \\
\mu_{X}(y)=0 \quad \text { if } y \text { is not in } X, \\
0<\mu_{X}(y)<1 \quad \text { if } y \text { is partly in } X .
\end{array}
$$

The above set explains the membership (characteristic) functions of $X$ which has the value ranges from 0 to 1 . It allows a wide range of possible values. The value from 0 to 1 in this set represents the degree of membership of element $y$ in set $X$. The membership function is commonly illustrated in terms of membership curve.

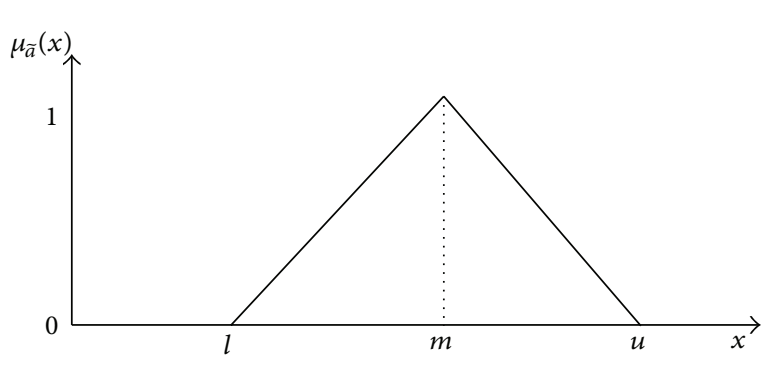

FIgURE 4: Symmetry TFN.

2.1. Triangular Fuzzy Number (TFN). There are 2 types of TFN which are symmetry and unsymmetry. Symmetry TFN is used in this paper since it enables users to easily calculate, understand, and capture the vagueness in people's verbal assessments [16].

TFN can be defined in three numbers, $(l, m, u)$ which represented the smallest possible value, the most promising value, and the largest value which describes the fuzzy event. This representation can be observed from Figure 4 which illustrates symmetry TFN [17].

Throughout this study, the commonly used algebraic operations for fuzzy numbers are addition and multiplication. The fuzzy operators shown below were adapted from [18]. Let $A$ and $B$ be two triangular fuzzy numbers with their parameters shown as follows:

$$
A=\left(a_{1}, a_{2}, a_{3}\right), \quad B=\left(b_{1}, b_{2}, b_{3}\right) .
$$

Fuzzy numbers addition is defined by

$$
A+B=\left(a_{1}+b_{1}, a_{2}+b_{2}, a_{3}+b_{3}\right) .
$$

On the other hand, fuzzy numbers multiplication is calculated as shown below:

$$
A \times B \approx\left(a_{1} b_{1}, a_{2} b_{2}, a_{3} b_{3}\right) .
$$

2.2. AHP and Fuzzy AHP. The analytical hierarchy process (AHP) was first developed by Saaty, in mid of 1970 [19]. It is a decision analysis method that considers both qualitative and quantitative attributes in decision making. It decomposes complex problem into tangible and intangible entities, transforms them into systematic hierarchies of criteria and alternatives, and ranks them in order from most to least importance or desirable. As such the complex problem can easily be comprehended and each level can be analyzed independently. Each criterion from the AHP structure will be compared against a given alternatives, thus, allowing it to judge the intensity of the importance of one criterion over the other based on the given alternatives [19].

AHP is simple, systematic, and a very useful approach which integrates the matrix theory. In defining the weights of criteria and comparing the alternatives, a set of pair-wise comparison has been developed by Saaty [8] as part of the steps in AHP. Since the concept of AHP considers only real exact number, the set of pair-wise comparison by Saaty has 
TABLE 4: Linguistic variables for importance of the criteria.

\begin{tabular}{lc}
\hline Linguistic variables & Scale of fuzzy number \\
\hline Very low (VL) & $(0,0,0.1,0.2)$ \\
Low $(\mathrm{L})$ & $(0.1,0.2,0.2,0.3)$ \\
Medium low $(\mathrm{ML})$ & $(0.2,0.3,0.4,0.5)$ \\
Medium $(\mathrm{M})$ & $(0.4,0.5,0.5,0.6)$ \\
Medium high $(\mathrm{MH})$ & $(0.5,0.6,0.7,0.8)$ \\
High $(\mathrm{H})$ & $(0.7,0.8,0.8,0.9)$ \\
Very high $(\mathrm{VH})$ & $(0.8,0.9,1.0,1.0)$ \\
\hline
\end{tabular}

TABLE 5: Linguistic variables for performance of the alternatives.

\begin{tabular}{lc}
\hline Linguistic variables & Scale of fuzzy number \\
\hline Very poor (VP) & $(0,0,1,2)$ \\
Poor (P) & $(1,2,2,3)$ \\
Medium poor (MP) & $(2,3,4,5)$ \\
Fair (F) & $(4,5,5,6)$ \\
Medium good (MG) & $(5,6,7,8)$ \\
Good (G) & $(7,8,8,9)$ \\
Very good (VG) & $(8,9,10,10)$ \\
\hline
\end{tabular}

been modified to suit human's judgement. This is the main reason, and the scale of weight and criteria preferences are defined in terms of linguistic variables in fuzzy numbers as presented in Tables 4 and 5 [2]. Linguistic variables are used to evaluate and assess the ratings of suppliers and weight of criteria. It is defined as variables with values expressed by words in a natural language [30].

There are three principles used in solving problems with AHP [9] in supplier selection which are as follows.

(i) AHP establishes the priorities based on sets of pairwise comparisons.

(ii) AHP score is built on human attributes and judgements where the intensity of each attribute or judgment is set according to its hierarchy over the other.

(iii) AHP synthesizes these judgments by using the hierarchy framework to obtain the overall priority of the elements or factors.

The concept of AHP and FAHP is mainly the same, and the difference of FAHP is that it analyses the numbers in terms of fuzzy numbers but as for AHP the numbers analyzed are crisp numbers. FAHP was firstly introduced by Van Laarhoven and Pedrycz in 1983 [31] whereby the fuzzy judgment is represented by triangular fuzzy number.

There are 6 steps in the process of decision making using FAHP. These steps are as follows.

(1) Firstly, form a decision matrix of the importance of each criterion with respect to each other.

(2) The membership function of triangular fuzzy number is defined by three real numbers $(l, m, u)$ which is mathematically described as $[\mu A(x)]$. The comparison matrix is derived from (6) as

$$
\begin{array}{r}
\mu_{A}(x)= \begin{cases}\frac{(x-l)}{m-l}, & l \leq x \leq m \\
\frac{u-x}{u-m}, & m \leq x \leq u \\
0, & \text { otherwise, }\end{cases} \\
a_{i j}=\frac{1}{a_{i j}}\left\{\begin{array}{c}
a_{i j}=\left(l_{i j}, m_{i j}, u_{i j}\right) \\
a_{i j}=\left(\frac{1}{u_{i j}}, \frac{1}{m_{i j}}, \frac{1}{l_{i j}}\right) .
\end{array}\right.
\end{array}
$$

(3) Calculate the $\widehat{G}_{i}$ (objective) which is defined by Chang in 1992 [32] in his study of fuzzy extent analysis. The computations of $\widehat{G}_{i}$ are explained in (7) as

$$
\begin{gathered}
\widehat{G}_{i}=\left(l_{i}, m_{i}, u_{i}\right), \\
l_{i}=\left(l_{i 1} \otimes l_{i 2} \otimes \cdots \otimes l_{i k}\right)^{1 / k} \quad i=1,2, \ldots, k \\
m_{i}=\left(m_{i 1} \otimes m_{i 2} \otimes \cdots \otimes m_{i k}\right)^{1 / k} \quad i=1,2, \ldots, k \\
u_{i}=\left(u_{i 1} \otimes u_{i 2} \otimes \cdots \otimes u_{i k}\right)^{1 / k} \quad i=1,2, \ldots, k .
\end{gathered}
$$

(4) The next step is the calculation of $\widehat{G}_{T}$ (total objective) whereby the equation used is displayed in (8) as

$$
\widehat{G}_{T}=\left(\sum_{i=1}^{k} l_{i}, \sum_{i=1}^{k} m_{i}, \sum_{i=1}^{k} u_{i}\right) .
$$

(5) Upon getting the result for $\widehat{G}_{T}$, weight of each criterion with respect to the objective is calculated. The equations used are shown in (9) as

$$
\begin{aligned}
\widehat{w} & =\frac{\widehat{G}_{i}}{\widehat{G}_{T}}=\frac{\left(l_{i}, m_{i}, u_{i}\right)}{\left(\sum_{i=1}^{k} l_{i}, \sum_{i=1}^{k} m_{i}, \sum_{i=1}^{k} u_{i}\right)} \\
& =\left[\frac{l_{i}}{\sum_{i=1}^{k} u_{i}}, \frac{m_{i}}{\sum_{i=1}^{k} m_{i}}, \frac{u_{i}}{\sum_{i=1}^{k} l_{i}}\right] .
\end{aligned}
$$

(6) The final stage is defuzzification whereby triangular fuzzy numbers are transformed into real numbers which is defined as weights $\left(W_{\text {in }}\right)$ and can be computed in (10) as

$$
W_{i n}=\frac{w_{i d}}{\sum_{i=1}^{k} w_{i d}}, \quad i=1,2, \ldots k
$$

The steps of 1 to 6 will be repeated for each alternative in terms of the criterion which will be called as the weight of factors in terms of alternatives. 
2.3. TOPSIS and Fuzzy TOPSIS. TOPSIS has been used in various fields and a number of applications such as outsourcing of logistics service, weapon selection, supplier selection analyzing business competition, and many other applications $[24,25,33]$. This method considers three types of criteria which are cost, qualitative, and quantitative. Generally, there are five steps in solving a problem using TOPSIS [34]. These steps are as follows:

(1) Obtain normalized decision matrix for $m$ alternatives over $n$ criteria. Let $x_{i j}$ score of option $i$ with respect to criterion $j$. Data can be normalized as below:

$$
r_{i j}=\frac{x_{i j}}{\left(\sum x^{2}{ }_{i j}\right)},
$$

for $i=1, \ldots, m$ and $j=1, \ldots, n$.

(2) Construct weighted normalized decision matrix, $v_{i j}$,

$$
v_{i j}=w_{j} \times r_{i j}
$$

whereby $w_{j}$ is a set of weights for each criteria and $r_{i j}$ is the normalized decision matrix.

(3) Identify the ideal alternative (extreme performance on each criterion, $A^{+}$) and negative ideal alternative (reverse extreme performance on each criterion, $A^{-}$) as

$$
A^{+}=\left\{v_{1}^{+}, \ldots, v_{n}^{+}\right\},
$$

whereby $v_{1}^{+}=\left\{\max _{i}\left(v_{i j}\right)\right.$ if $j \in J^{+} ; \min _{i}\left(v_{i j}\right)$ if $\left.j \in J^{-}\right\}$ and

$$
A^{-}=\left\{v_{1}^{-}, \ldots, v_{n}^{-}\right\}
$$

whereby $v_{1}^{-}=\left\{\min _{i}\left(v_{i j}\right)\right.$ if $j \in J^{-} ; \min _{i}\left(v_{i j}\right)$ if $\left.j \in J^{-}\right\}$.

(4) Develop a distance measure over criterion to both ideal $\left(D^{+}\right)$and negative ideal alternative $\left(D^{-}\right)$as

$D_{i}^{+}=\left[\Sigma\left(v_{j}^{+}-v_{i j}\right)^{2}\right]^{1 / 2}, \quad D^{-}=\left(\Sigma\left(v_{j}^{-}-v_{i j}\right)^{2}\right)^{1 / 2}$.

(5) For each alternative, determine the relative closeness to the ideal solution, $C_{i}^{+} . C_{i}^{+}$is equal to the distance of the negative ideal solution divided by the sum of the distance of the negative ideal and ideal solution given by

$$
C_{i}^{+}=\frac{D^{-}}{D^{-}+D^{+}}
$$

TOPSIS only considers crisp values, whereas human judgments are usually uncertain and could not be evaluated using fix numbers. In spite of this, fuzzy numbers are used to replace all the crisp values in TOPSIS. In decision making, it is difficult to give a certain judgement, hence by integrating fuzzy logic and TOPSIS it will eliminate the uncertainty of the decision made [35]. Fuzzy TOPSIS has been successfully applied in several MCDM problems [36-39].

In performing FTOPSIS, Chen in 2000 [36] underlined 9 steps to be followed. The steps of the algorithm in this multicriteria decision making tool are as follows.
TABLE 6: Linguistic variables for the importance weight of each criterion.

\begin{tabular}{lc}
\hline Linguistic variables & Fuzzy value \\
\hline Very low (VL) & $(0,0,0.1)$ \\
Low $(\mathrm{L})$ & $(0,0.1,0.3)$ \\
Medium low $(\mathrm{ML})$ & $(0.1,0.3,0.5)$ \\
Medium high $(\mathrm{MH})$ & $(0.5,0.7,0.9)$ \\
High $(\mathrm{H})$ & $(0.7,0.9,1.0)$ \\
Very high $(\mathrm{VH})$ & $(0.9,1.0,1.0)$ \\
\hline
\end{tabular}

TABLE 7: Linguistic variables for the ratings.

\begin{tabular}{lc}
\hline Linguistic variables & Fuzzy value \\
\hline Very poor (VP) & $(0,0,1)$ \\
Poor (P) & $(0,1,3)$ \\
Medium poor (MP) & $(1,3,5)$ \\
Fair (F) & $(3,5,7)$ \\
Medium good (MG) & $(5,7,9)$ \\
Good (G) & $(7,9,10)$ \\
Very good (VG) & $(9,10,10)$ \\
\hline
\end{tabular}

Step 1. A committee of decision makers were formed to evaluate the alternatives based on the goal defined using the linguistic variables in Table 6.

Step 2. Appropriate linguistic variables for the ratings of the alternative with respect to criteria were chosen and these linguistic ratings used are taken from Table 7 [36].

Step 3. The importance weights of criteria and the ratings of three candidates are converted into fuzzy values. In order to obtain the fuzzy decision matrix and fuzzy weights of three alternatives, average value of each criteria for 3 decision makers was taken.

Step 4. The next step of the FTOPSIS analysis is the normalization of fuzzy decision matrix. This step is performed by taking each respective value and divide it with the maximum number of the particular criteria for all the 3 candidates.

Step 5. Construction of fuzzy weighted normalized decision matrix was then generated. In order to generate these values, values in Step 4 were divided by the weight of the respective column of fuzzy weighted normalized decision matrix.

Step 6. Fuzzy positive ideal solution (FPIS) and fuzzy negative ideal solution (FNIS) were then defined based on the values of normalized positive and negative triangular fuzzy numbers.

Step 7. Distance of each alternative from FPIS and FNIS was then calculated. The distance of two fuzzy numbers is calculated in (17) as

$$
\sqrt{\frac{1}{3}\left[\left(m_{1}-n_{1}\right)^{2}+\left(m_{2}-n_{2}\right)^{2}+\left(m_{3}-n_{3}\right)^{2}\right]} .
$$


TABLE 8: Summary of advantages of MCDM models.

Differences

AHP FAHP TOPSIS FTOPSIS FAHPiFTOPSIS

Evaluators are able to represent the relative importance and interaction of multiple criteria in the supplier selection process [20]

Bias in decision making can be reduced by the flexibility and ability to check on inconsistency and able to decompose and problems into hierarchies of criteria. [21]

Accurate, effective, and systematic decision support tool [22]

Effectively handle both qualitative and quantitative data and easy to implement and understand $[23,24]$

No tedious pairwise comparison and weights can be directly assigned by decision makers which makes the practical application of the methodology very straightforward [22, 25]

TOPSIS has been proved to be one of the best methods addressing rank reversal issue, that is, the change in the ranking of the alternatives when a nonoptimal alternative is introduced [22]

Fuzzy AHP is preferable for widely spread hierarchies, where few importance/rating pair-wise comparisons are required at lower level trees [22]

Can adopt linguistic variables [22]

By using fuzzy AHP and fuzzy TOPSIS, uncertainty and vagueness from subjective perception and the experiences of decision maker can be effectively represented and reached to a more effective decision [22]

Ranking results for both methods are similar which shows that when decision makers are consistent in determining the data, two methods independently, and the ranking results will be the same and will handle fuzziness of data involved in decision making $\mathrm{Y}$

$\mathrm{Y}$ $\mathrm{Y}$ effectively [22]

Note: $\mathrm{Y}$ means the differences are applicable for the respective MCDM.

TABLE 9: Summary of disadvantages of MCDM models.

Differences

When a problem is decomposed into subsystems, the decision problem might become very large and lengthy [7]

AHP's using crisp number, hence not able to reflect human thinking style [7]

When a number of alternatives and criteria increased, pair-wise comparison becomes cumbersome and risk of inconsistencies grows [22, 25-27]

Problem is not decomposed into hierarchy hence decision maker might encounter difficulty to simplify the problem

Integration with FAHP resulted in a number of extra steps to be followed

Does not take into account the uncertainty associated with the mapping of one's judgment to a number [23]

FAHP requires more complex computations than FTOPSIS which includes pair wise comparison $[22,28]$

In the extent analysis of FAHP, the priority weights of criterion or alternative can be equal to zero [22]

Note: Y means the differences are applicable for the respective MCDM.

Step 8. After complete computing the positive and negative distance, these values were then summed up to obtain the total distance measurement for positive values, $A^{*}$, and negative values, $A^{-}$.

Step 9. Finally, obtain the final ranking of the alternative closeness coefficient, CC. In order to achieve the CC, (18) is used as

$$
\frac{d_{i}^{-}}{d_{i}^{-}+d_{i}^{+}}
$$

As per quoted by Krohling and Campanharo in 2011 [35], the triangular fuzzy number used is very effective for solving decision making problem which involved uncertainty and imprecise judgement.

2.4. Fuzzy AHP Integrated with Fuzzy TOPSIS (FAHPiFTOPSIS). Integration between fuzzy AHP and fuzzy TOPSIS is believed to be able to make decision making more practical and reliable for decision makers due to its human-like thinking capability. For FAHPiFTOPSIS, the process of decision making comprises of 9 steps $[2,30]$. 
TABLE 10: Weight and level of importance of criteria 1 to 9 of 12 . Major automotive manufacturers.

\begin{tabular}{|c|c|c|c|}
\hline Criteria & Level of importance & Respondent & Mean \\
\hline \multicolumn{4}{|l|}{ (a) Delivery/lead time (DT) } \\
\hline (1) On time delivery & 58 & 12 & 4.83 \\
\hline (2) No shipping error (incorrect shipment) & 53 & 12 & 4.42 \\
\hline (3) Able to deliver supplies within short lead time & 50 & 12 & 4.17 \\
\hline (4) Products delivered in good conditions & 56 & 12 & 4.67 \\
\hline (5) Proper delivery record and followup & 58 & 12 & 4.83 \\
\hline (6) Applies JIT (just in time) concept & 50 & 12 & 4.17 \\
\hline Total & 325 & 72 & \\
\hline Weightage (mean) & 4.5139 & $\sigma$ & 0.31 \\
\hline Weightage (mean) (\%) & 11.7247 & Var & 0.10 \\
\hline Importance weight & 0.1175 & & \\
\hline \multicolumn{4}{|l|}{ (b) Support service (SS) } \\
\hline (1) Handled by supplier technical experts & 56 & 12 & 4.67 \\
\hline (2) Supplier support readily available & 57 & 12 & 4.75 \\
\hline (3) Promptness of response & 56 & 12 & 4.67 \\
\hline Total & 169 & 36 & \\
\hline Weightage (mean) & 4.6944 & $\sigma$ & 0.05 \\
\hline Weightage (mean) (\%) & 12.1937 & Var & 0.002 \\
\hline Importance weight & 0.12194 & & \\
\hline \multicolumn{4}{|l|}{ (c) Quality factor (QF) } \\
\hline (1) Meeting customer requirement and expectations & 55 & 12 & 4.58 \\
\hline (2) Reliability in supply quality & 58 & 12 & 4.83 \\
\hline (3) Product certification & 55 & 12 & 4.58 \\
\hline (4) Provide sample of supply before first ordering & 53 & 12 & 4.42 \\
\hline (5) Proper record on complaints and followup & 55 & 12 & 4.58 \\
\hline (6) Raw material quality and supply reliability & 57 & 12 & 4.75 \\
\hline (7) Special packaging and shipping requirement & 51 & 12 & 4.25 \\
\hline (8) Proper marking and labeling of materials & 54 & 12 & 4.50 \\
\hline (9) Conformance to environmental standard specifications & 53 & 12 & 4.42 \\
\hline (10) Safety and health record performance & 53 & 12 & 4.42 \\
\hline Total & 544 & 120 & \\
\hline Weightage (mean) & 4.5333 & $\sigma$ & 0.17 \\
\hline Weightage (mean) (\%) & 11.7753 & Var & 0.03 \\
\hline Importance weight & 0.11775 & & \\
\hline \multicolumn{4}{|l|}{ (d) Technology (TE) } \\
\hline (1) Availability of production facilities and capacity & 56 & 12 & 4.67 \\
\hline (2) Technological capabilities for future improvement & 48 & 12 & 4.00 \\
\hline (3) Innovativeness in product design and development & 47 & 12 & 3.92 \\
\hline (4) Technical support on product development & 51 & 12 & 4.25 \\
\hline (5) Intellectual property & 45 & 12 & 3.75 \\
\hline (6) Information system capability (ICT, EDI, ERP, barcode) & 46 & 12 & 3.83 \\
\hline (7) Shorter product development lead time & 50 & 12 & 4.17 \\
\hline Total & 343 & 84 & \\
\hline Weightage (mean) & 4.0833 & $\sigma$ & 0.31 \\
\hline Weightage (mean) (\%) & 10.6064 & Var & 0.10 \\
\hline Importance weight & 0.10606 & & \\
\hline
\end{tabular}


TABle 10: Continued.

\begin{tabular}{|c|c|c|c|}
\hline Criteria & Level of importance & Respondent & Mean \\
\hline \multicolumn{4}{|l|}{ (e) Price/cost (PR) } \\
\hline (1) Competitive pricing & 56 & 12 & 4.67 \\
\hline (2) Additional discount for large volume purchase & 52 & 12 & 4.33 \\
\hline (3) No charges to distribution/logistic costs & 44 & 12 & 3.67 \\
\hline (4) No charges after sales services costs & 46 & 12 & 3.83 \\
\hline (5) No additional costs for small volume order & 46 & 12 & 3.83 \\
\hline Total & 244 & 60 & \\
\hline Weightage (mean) & 4.0667 & $\sigma$ & 0.42 \\
\hline Weightage (mean) (\%) & 10.5631 & Var & 0.18 \\
\hline Importance weight & 0.10563 & & \\
\hline \multicolumn{4}{|l|}{ (f) Factory capacity and capability (FC) } \\
\hline (1) High production capacity & 51 & 12 & 4.25 \\
\hline (2) Capability to cope with any order changes & 49 & 12 & 4.08 \\
\hline (3) Maintain workforce competency & 49 & 12 & 4.08 \\
\hline (4) Reliable production facilities & 54 & 12 & 4.50 \\
\hline (5) Reliable maintenance programme & 52 & 12 & 4.33 \\
\hline (6) Apply OEE concept & 51 & 12 & 4.25 \\
\hline Total & 306 & 72 & \\
\hline Weightage (mean) & 4.2500 & $\sigma$ & 0.16 \\
\hline Weightage (mean) (\%) & 11.0393 & Var & 0.03 \\
\hline Importance weight & 0.11039 & & \\
\hline
\end{tabular}

(g) Supplier background (SB)

(1) Industry and technological knowledge on product

$\begin{array}{ccc}54 & 12 & 4.50 \\ 48 & 12 & 4.00 \\ 52 & 12 & 4.33 \\ 50 & 12 & 4.17 \\ 49 & 12 & 4.08 \\ 50 & 12 & 4.17 \\ 303 & 72 & \\ 4.2083 & \sigma & 0.18 \\ 10.9311 & \text { Var } & 0.03 \\ 0.10931 & & \end{array}$

(2) Having own transportation

(3) Strong financial management and support

(4) Approved supplier to other established Tier 1 or 2 company

(5) Available supply of skilled workforce

(6) Strategic geographical location of the supplier

Total

Weightage (mean)

Weightage (mean) (\%)

0.10931

Importance weight

(h) Flexibility (FL)

(1) Flexible manufacturing system

\begin{tabular}{ccc}
47 & 12 & 3.92 \\
50 & 12 & 4.17 \\
45 & 12 & 3.75 \\
48 & 12 & 4.00 \\
190 & 48 & \\
\hline 3.9583 & $\sigma$ & 0.17 \\
10.2817 & Var & 0.30 \\
0.10282 & & \\
\hline
\end{tabular}

(2) Flexibility in operation and production 50

(3) Flexibility in product design changes

(4) Flexibility to respond on changes in requirement

Total

Weightage (mean)

Weightage (mean) (\%)

0.10282

Importance weight

(i) Other management system requirements (MS)
(1) Kanban
(2) Kaizen
(3) TPS (Toyota production system)
(4) Six sigma
(5) 5 S implementation
(6) Failure modes and effect analysis (FMEA) 
TABLE 10: Continued.

\begin{tabular}{lcc}
\hline Criteria & Level of importance & Respondent \\
\hline (7) Statistical process control (SPC) & 46 & 12 \\
(8) Production part approval process (PPAP) & 50 & 3.83 \\
(9) Advanced product quality planning (APQP) & 45 & 4.17 \\
(10) Measurement systems analysis (MSA) & 44 & 12 \\
(11) TS16949 technical specification & 56 & 12 \\
(12) ISO 9001 quality management system & 56.75 \\
(13) ISO 14001 environmental management system & 3.67 \\
(14) OHSAS 18001 safety and health management system & 54 & 12 \\
\hline Total & 53 & 12 \\
\hline Weightage (mean) & 704 & 12 \\
Weightage (mean) (\%) & 4.67 \\
Importance weight & 4.1905 & 12 \\
\hline Grand total weightage (mean) & 10.8847 & \\
\hline
\end{tabular}

(1) Construct pair-wise comparison matrices among all the elements or criteria in the dimensions of the hierarchy system and convert it into fuzzy.

(2) Define the fuzzy geometric mean as

$$
\tilde{r}_{i}=\left(\tilde{a}_{i 1} \otimes \cdots \otimes \tilde{a}_{i j} \otimes \cdots \otimes \tilde{a}_{i n}\right)^{1 / n} .
$$

(3) Define the fuzzy weights as

$$
\widetilde{w}_{i}=\tilde{r} \otimes\left[\widetilde{r}_{i} \oplus \cdots \oplus \widetilde{r}_{i} \oplus \cdots \oplus \widetilde{r}_{n}\right]^{-1} .
$$

(4) Aggregating the ranking of criteria and suppliers' performance criteria ranking as

$$
\tilde{x}_{i j k}=\left(a_{i j k}, b_{i j k}, c_{i j k}\right) \text {, }
$$

where

$$
\begin{gathered}
a_{i j}=\min _{k}\left\{a_{i j k}\right\}, \\
b_{i j}=\frac{1}{k} \sum_{k=1}^{k} b_{i j k}, \\
c_{i j}=\max _{k}\left\{c_{i j k}\right\} .
\end{gathered}
$$

(5) Suppliers' performance as

$$
\widetilde{w}_{j}=\left(w_{j 1}, w_{j 2}, w_{j 3}\right),
$$

where

$$
\begin{aligned}
& w_{j 1}=\min _{k}\left\{w_{j k 1}\right\}, \\
& w_{j 2}=\frac{1}{k} \sum_{k=1}^{k} w_{j k 2}, \\
& w_{j 3}=\max _{k}\left\{w_{j k 3}\right\} .
\end{aligned}
$$

(6) Normalizing the fuzzy decision matrix as

$$
\widetilde{R}=\left[\widetilde{r}_{i j}\right]_{m \times n}, \quad i=1,2, \ldots m, j=1,2, \ldots n,
$$

where

$$
\begin{gathered}
\tilde{r}_{i j}=\left(\frac{a_{i j}}{c_{j}^{*}}, \frac{b_{i j}}{c_{j}^{*}}, \frac{c_{i j}}{c_{j}^{*}}\right), \\
c_{j}^{*}=\max _{i} c_{i j} .
\end{gathered}
$$

(7) Weighted normalization of the fuzzy decision matrix as

$\widetilde{V}=\left[\widetilde{v}_{i j}\right]_{m \times n}, \quad i=1,2, \ldots m, j=1,2, \ldots n$

where

$$
\widetilde{v}_{i j}=\widetilde{r}_{i j}(\bullet) \widetilde{w}_{j}
$$

(8) Distance to positive and negative ideal solution using vertex method [36]. Let $\widetilde{m}=\left(m_{1}, m_{2}, m_{3}\right)$ and $\widetilde{n}=$ $\left(n_{1}, n_{2}, n_{3}\right)$ between two triangular fuzzy numbers is as

$d_{v}(\widetilde{m}, \widetilde{n})=\sqrt{\frac{1}{3}\left[\left(m_{1}-n_{1}\right)^{2}+\left(m_{2}-n_{2}\right)^{2}+\left(m_{3}-n_{3}\right)^{2}\right.}$.

The best level of solution denoted by $A^{+}$and the worst level of solution denoted by $A^{-}$are defined in (30) as

$$
\begin{aligned}
& A^{+}=\left(\widetilde{v}_{1}^{*}, \widetilde{v}_{2}^{*}, \ldots, \widetilde{v}_{n}^{*}\right), \\
& A^{-}=\left(\widetilde{v}_{1}^{-}, \widetilde{v}_{2}^{-}, \ldots, \widetilde{v}_{n}^{-}\right),
\end{aligned}
$$


TABLE 11: Weight and level of importance of nine (9) criteria of 4 major tyre suppliers.

\begin{tabular}{|c|c|c|c|}
\hline Criteria & Level of importance & Respondent & Mean \\
\hline \multicolumn{4}{|l|}{ (a) Delivery/lead time (DT) } \\
\hline (1) On time delivery & 19 & 4 & 4.75 \\
\hline (2) No shipping error (incorrect shipment) & 17 & 4 & 4.25 \\
\hline (3) Able to deliver supplies within short lead time & 15 & 4 & 3.75 \\
\hline (4) Products delivered in good conditions & 19 & 4 & 4.75 \\
\hline (5) Proper delivery record and followup & 19 & 4 & 4.75 \\
\hline (6) Applies JIT (just in time) concept & 16 & 4 & 4 \\
\hline Total & 105 & 24 & \\
\hline Weightage (mean) & 4.3750 & $\sigma$ & 0.44 \\
\hline Weightage (mean) (\%) & 11.6600 & Var & 0.19 \\
\hline Importance weight & 0.11660 & & \\
\hline \multicolumn{4}{|l|}{ (b) Support service (SS) } \\
\hline (1) Handled by supplier technical experts & 19 & 4 & 4.75 \\
\hline (2) Supplier support readily available & 17 & 4 & 4.25 \\
\hline (3) Promptness of response & 18 & 4 & 4.5 \\
\hline Total & 54 & 12 & \\
\hline Weightage (mean) & 4.5000 & $\sigma$ & 0.25 \\
\hline Weightage (mean) (\%) & 11.9931 & Var & 0.06 \\
\hline Importance weight & 0.11993 & & \\
\hline
\end{tabular}

(c) Quality factor (QF)

(1) Meeting customer requirement and expectations $\quad 19 \quad 4 \quad 4.75$

(2) Reliability in supply quality $\quad 19 \quad 4.75$

(3) Product certification $\quad 15 \quad 3.75$

(4) Provide sample of supply before first ordering $\quad 15 \quad 4.75$

(5) Proper record on complaints and followup $\quad 15 \quad 4.75$

(6) Raw material quality and supply reliability $\quad 19 \quad 4.75$

(7) Special packaging and shipping requirement $\quad 14 \quad 4.5$

(8) Proper marking and labeling of materials $\quad 15 \quad 4.75$

(9) Conformance to environmental standard specifications $\quad 15 \quad 4.75$

(10) Safety and health record performance $\quad 17 \quad 4 \quad 4.25$

\begin{tabular}{|c|c|c|c|}
\hline Total & 163 & 40 & \\
\hline Weightage (mean) & 4.0750 & $\sigma$ & 0.50 \\
\hline Weightage (mean) (\%) & 10.8605 & Var & 0.25 \\
\hline Importance weight & 0.10860 & & \\
\hline
\end{tabular}

(d) Technology (TE)

(1) Availability of production facilities and capacity $\quad 16 \quad 4 \quad 4$

(2) Technological capabilities for future improvement $\quad 16 \quad 4 \quad 4$

(3) Innovativeness in product design and development $\quad 16 \quad 4 \quad 4$

(4) Technical support on product development $\quad 19 \quad 4.75$

(5) Intellectual property $\quad 12 \quad 4 \quad 3$

(6) Information system capability (ICT, EDI, ERP, barcode) $\quad 15 \quad 4.75$

(7) Shorter product development lead time $\quad 16 \quad 4 \quad 4$

\begin{tabular}{|c|c|c|c|}
\hline Total & 110 & 28 & \\
\hline Weightage (mean) & 3.9286 & $\sigma$ & 0.51 \\
\hline Weightage (mean) (\%) & 10.4702 & Var & 0.26 \\
\hline Importance weight & 0.10470 & & \\
\hline
\end{tabular}


TABLE 11: Continued.

\begin{tabular}{|c|c|c|c|}
\hline Criteria & Level of importance & Respondent & Mean \\
\hline \multicolumn{4}{|l|}{ (e) Price/cost (PR) } \\
\hline (1) Competitive pricing & 18 & 4 & 4.5 \\
\hline (2) Additional discount for large volume purchase & 15 & 4 & 3.75 \\
\hline (3) No charges to distribution/logistic costs & 13 & 4 & 3.25 \\
\hline (4) No charges after sales services costs & 13 & 4 & 3.25 \\
\hline (5) No additional costs for small volume order & 16 & 4 & 4 \\
\hline Total & 75 & 20 & \\
\hline Weightage (mean) & 3.7500 & $\sigma$ & 0.53 \\
\hline Weightage (mean) (\%) & 9.9943 & Var & 0.28 \\
\hline Importance weight & 0.09994 & & \\
\hline \multicolumn{4}{|l|}{ (f) Factory capacity and capability (FC) } \\
\hline (1) High production capacity & 16 & 4 & 4.00 \\
\hline (2) Capability to cope with any order changes & 16 & 4 & 4.00 \\
\hline (3) Maintain workforce competency & 17 & 4 & 4.25 \\
\hline (4) Reliable production facilities & 19 & 4 & 4.75 \\
\hline (5) Reliable maintenance programme & 17 & 4 & 4.25 \\
\hline (6) Apply OEE concept & 15 & 4 & 3.75 \\
\hline Total & 100 & 24 & \\
\hline Weightage (mean) & 4.1667 & $\sigma$ & 0.34 \\
\hline Weightage (mean) (\%) & 11.1048 & Var & 0.12 \\
\hline Importance weight & 0.11105 & & \\
\hline
\end{tabular}

(g) Supplier background (SB)

(1) Industry and technological knowledge on product $\quad 16 \quad 4 \quad 4$

(2) Having own transportation $\quad 15 \quad 4.75$

(3) Strong financial management and support $\quad 19 \quad 4 \quad 4.75$

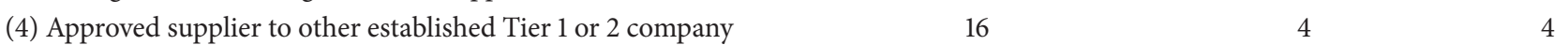

(5) Available supply of skilled workforce $\quad 16 \quad 4 \quad 4$

(6) Strategic geographical location of the supplier $\quad 16 \quad 4 \quad 4$

\begin{tabular}{|c|c|c|c|}
\hline Total & 98 & 24 & \\
\hline Weightage (mean) & 4.0833 & $\sigma$ & 0.34 \\
\hline Weightage (mean) (\%) & 10.8827 & Var & 0.12 \\
\hline Importance weight & 0.10883 & & \\
\hline
\end{tabular}

(h) Flexibility (FL)

(1) Flexible manufacturing system $\quad 17 \quad 4 \quad 4.25$

(2) Flexibility in operation and production $\quad 18 \quad 4 \quad 4.50$

(3) Flexibility in product design changes $\quad 17 \quad 4 \quad 4.25$

(4) Flexibility to respond on changes in requirement $\quad 18 \quad 4 \quad 4.50$

\begin{tabular}{|c|c|c|c|}
\hline Total & 70 & 16 & \\
\hline Weightage (mean) & 4.3750 & $\sigma$ & 0.14 \\
\hline Weightage (mean) (\%) & 11.6600 & Var & 0.02 \\
\hline Importance weight & 0.11660 & & \\
\hline
\end{tabular}

(i) Other management system requirements (MS)

$\begin{array}{llll}\text { (1) Kanban } & 17 & 4 & 4.25 \\ \text { (2) Kaizen } & 18 & 4 & 4.50 \\ \text { (3) TPS (Toyota production system) } & 17 & 4.25 \\ \text { (4) Six sigma } & 16 & 4 & 4.00 \\ \text { (5) } 5 \text { S implementation } & 19 & 4 & 4.75 \\ \text { (6) Failure modes and effect analysis (FMEA) } & 16 & 4 \\ \text { (7) Statistical process control (SPC) } & 15 & 4.00 \\ \end{array}$


TABLE 11: Continued.

\begin{tabular}{lccr}
\hline Criteria & Level of importance & Respondent & Mean \\
\hline (8) Production part approval process (PPAP) & 17 & 4 & 4.25 \\
(9) Advanced product quality planning (APQP) & 18 & 4 & 4.50 \\
(10) Measurement systems analysis (MSA) & 18 & 4 & 4.50 \\
(11) TS16949 technical specification & 17 & 4 & 4.25 \\
(12) ISO 9001 quality management system & 17 & 4 & 4.25 \\
(13) ISO 14001 environmental management system & 17 & 4 & 4.25 \\
(14) OHSAS 18001 safety and health management system & 17 & 4 & 4.25 \\
\hline Total & 239 & & 0.25 \\
\hline Weightage (mean) & 4.2679 & 0.06 \\
Weightage (mean) (\%) & 11.3745 & \\
Importance weight & 0.11374 & & \\
\hline Grand total weightage (mean) & 37.5214 & & \\
\hline
\end{tabular}

where

$$
\begin{gathered}
\widetilde{v}_{j}^{*}=(1,1,1) \otimes \widetilde{w}_{j}=\left(l w_{j} ; m w_{j}, u w_{j}\right), \\
\widetilde{v}_{j}^{-}=(0,0,0), \quad j=1,2, \ldots, n .
\end{gathered}
$$

The distances $\left(d_{i}^{+}\right.$and $\left.d_{i}^{-}\right)$between suppliers from $A^{+}$ and $A^{-}$can be achieved by the area compensation method which is shown in the following equations:

$$
\begin{aligned}
& \tilde{d}_{i}^{+}=\sum_{j=1}^{n} d\left(\widetilde{v}_{i j}, \widetilde{v}_{j}^{*}\right), \quad i=1,2, \ldots, m ; j=1,2, \ldots, n, \\
& \widetilde{d}_{i}^{-}=\sum_{j=1}^{n} d_{v}\left(\widetilde{v}_{i j} \widetilde{v}_{j}^{-}\right), \quad i=1,2, \ldots, m ; j=1,2, \ldots, n .
\end{aligned}
$$

(9) Closeness coefficient as

$$
\widetilde{C} \widetilde{C}_{i}=\frac{\widetilde{d}_{i}^{-}}{\widetilde{d}_{i}^{+}+\widetilde{d}_{i}^{-}}, \quad i=1,2, \ldots, m .
$$

2.5. Summary of Advantages and Disadvantages of MCDM Models. All the 5 models mentioned above are basically focusing on the theoretical part of the decision making. In this subsection the models are compared with respect to the differences. Based on Table 8, every model has its advantages. However, there is one common advantage which caters for all 5 models which is the effectiveness to handle qualitative and quantitative data.

Table 8 summarized the advantages of 5 models of focus in this study. For FAHP, AHP, and FAHPiFTOPSIS, these models are able to decompose complex problems into hierarchy which simplify the process of decision making and reduce the biasness by checking the inconsistency of rating. This is different for TOPSIS and FTOPSIS whereby the weights defined by decision makers are directly inserted into the models without any pairwise comparison. Models incorporated with fuzzy logic such as FAHP, FTOPSIS, and
FAHPiFTOPSIS can adopt linguistic variables which convert expression of words to fuzzy numbers. FAHPiFTOPSIS is a versatile MCDM which adopted the decomposed hierarchy system of FAHP and the best method of ranking issue by FTOPSIS.

Referring to Table 9, it analysed the disadvantages of every model. These disadvantages can be compensated by combining the model with another MCDM model, for instance FAHPiFTOPSIS whereby FAHP is integrated with FTOPSIS.

Since FAHPiFTOPSIS integrated with FAHP and FTOPSIS, there are more steps that need to be performed. When comparing FAHP to FTOPSIS, FAHP requires more complex computation which includes pairwise comparison. When the number of alternatives and criteria increased, pairwise comparison becomes cumbersome. For AHP and FAHP besides the advantage of decomposing the problems, this might also lead to a large and lengthy process. AHP and TOPSIS are not integrated; hence, the models will not be able to reflect human thinking style.

\section{Research Methodology}

Companies involved in this study are automotive supply companies, Malaysian automotive manufacturers, and foreign automotive manufacturing plants operating in Malaysia together with the cooperation of four major tyre manufacturers in Malaysia. The process of the study started with the determination of the important criteria and their ranking which was made through surveys conducted on supply companies for automotive manufacturers. The data and information collected were analysed in depth and compared against traditional criteria.

Five methods of MCDM which are AHP, FAHP, TOPSIS, FTOPSIS, and an integrated FAHPiFTOPSIS model will be investigated. The effectiveness of all the five MCDM models will be compared. This study will be conducted through questionnaires distributed to Malaysian automotive manufacturers and foreign companies which supply preassembled 
parts to automotive manufacturers. The respondents of this study comprise 4 major tyre manufacturers and 12 automotive manufacturing plants.

3.1. Phase 1: Data Collection. This phase starts with the design of the data collection protocol, which concerns data to be collected and how to collect the data. The data used in this paper are collected and obtained from several sources such as questionnaires, face-to-face interviews, and phone interviews. The questionnaire aimed at identifying the priorities of various criteria highlighted in selecting suppliers as attached in Appendix C. In this phase, the interviews have been conducted by visiting the selected automotive industry. Phone interviews were also conducted whenever necessary. The purpose of this interview is to have a clearer picture on how supplier selection process is performed and at the same time to clarify any doubts regarding the questionnaires answered earlier. Nine main criteria were identified by the author for supplier selection process in automotive industry based on initial interview conducted with one of the major local car manufacturers. These criteria are shown in Appendix A.

\subsection{Phase 2: Analyzing and Comparing Supplier Selection} Models Using AHP, FAHP, TOPSIS, FTOPSIS, and FAHPiFTOPSIS. In this phase, real quantitative data are used. After identifying the criteria which are critical and important in selecting suppliers, the next stage of the project will be structuring the existing AHP, FAHP, TOPSIS, FTOPSIS, and FAHPiFTOPSIS models for supplier selection using data obtained from the questionnaire. Results obtained from the survey which consists of goal, criteria, subcriteria, and alternatives are used in structuring the models.

The models used for AHP and TOPSIS are from the founders themselves which are Saaty [8] and Hwang and Yoon 1981 [11], whereas for FAHP, the calculation method applied in this research is based on Chang's approach [32]. Chang introduced a new approach for handling the fuzzy AHP-the use of TFNs for a pairwise comparison scale of the fuzzy AHP and the use of the extent analysis method for the synthetic extent values of the pairwise comparisons. Chang's approach is one of the most popular approaches in the fuzzy AHP field. In this work, Chang's extent analysis method is preferred whereby the steps of this approach are relatively easier than the other fuzzy AHP approaches and are similar to the conventional AHP. Considering Buckley [40], the model developed utilizes trapezoidal calculation and is tremendous. For FTOPSIS, a general extension of TOPSIS in fuzzy environment has been developed by Chen 2000 [36] which is the model utilized in this study, whereas for FAHPiFTOPSIS the model used is by Sun 2010 [30].

Once these five models are structured, the results produced by the models are then analysed. The purpose of this analysis is to determine the qualities, shortcomings, and biasness of the models. For AHP, FAHP, and FAHPiFTOPSIS, bias in decision making can be eliminated due to its flexibility to check the inconsistency and ability to decompose problems into hierarchies [21]. To determine which model has the less variation which means less bias in selection process, computation of variation percentage between models is performed in the analysis section $[41,42]$.

3.3. Phase 3: Testing and Validation of the Models. The final step of this paper is to validate the models using existing data collected from the questionnaire. This is to prove the consistency of the results for the five models with the results of the supplier selection of in-house procedures of the companies under study. The results of the models are then analyzed with the current practice of the selected companies. The main purpose of this step is to test if the results are consistent with the models and also to observe the best method relevant to the companies in obtaining the best supplier for automotive industry.

The system architecture example of the models is illustrated in Appendix B which involves one decision maker, decision maker 1 (DM1), and three potential alternatives, supplier 1 (S1), supplier 2 (S2), and supplier 3 (S3) with 9 criteria adopting the triangular values of fuzzy. From the figure, it can be seen that the system has three major components which are

$$
\begin{aligned}
& \text { "A": selection of criteria ranking and supplier's per- } \\
& \text { formance, } \\
& \text { "B": interpretation of data, } \\
& \text { "C": results. }
\end{aligned}
$$

In the first component (A), decision maker is required to pairwise compare the suppliers' performance with respect to the criteria. Further ranking of the criteria based on its importance towards the goal is then performed. Accordingly, performance of the suppliers based on the criteria is then selected. In the second component (B), the interpretation of data takes place where all the data collected from the survey are inserted as input for all the models. All the calculation of analysis is done using Microsoft Excel 2010. The pairwise comparison and ranking utilized the linguistic variable method which are variables with values expressed by words in a natural language [30]. All the data inputs are selected based on the linguistic variable table provided. Component " $C$ " is the final major component of the example in obtaining the best supplier according to the desired criteria.

3.4. Application in Automotive Industries. In Tables 10 and 11, the data tabulated are the number of respondents and level of importance for criteria 1 to 9 responded by 12 major automotive manufacturers and 4 major tyre suppliers in Malaysia.

\section{Results and Discussion}

Upon analyzing the various decision making tools described in Section 3, Figure 5 is presented. The figure illustrates the overall ranking for 5 different decision making tools used for supplier selection process in automotive industries using the results tabulated in Table 12 .

Referring to Table 12, these are the values of actual scores for Supplier 1, 2, 3, and 4 according to the decision making 
TABle 12: Actual scores of Supplier 1,2, 3, and 4 according to 5 decision making tools.

\begin{tabular}{lccccc}
\hline & AHP & FAHP & TOPSIS & FTOPSIS & FAHPiFTOPSIS \\
\hline Supplier 1 & 0.16451 & 0.18905 & 0.23400 & 0.31199 & 0.25827 \\
Supplier 2 & 0.16414 & 0.11949 & 0.18632 & 0.29032 & 0.25693 \\
Supplier 3 & 0.43261 & 0.41440 & 0.98365 & 0.92561 & 0.48116 \\
Supplier 4 & 0.23874 & 0.25259 & 0.46652 & 0.49101 & 0.37255 \\
\hline
\end{tabular}

TABLE 13: Normalised scores of Supplier 1, 2, 3, and 4 according to 5 decision making tools.

\begin{tabular}{lccccc}
\hline & AHP & FAHP & TOPSIS & FTOPSIS & FAHPiFTOPSIS \\
\hline Supplier 1 & 0.16451 & 0.14389 & 0.12510 & 0.15453 & 0.18867 \\
Supplier 2 & 0.16414 & 0.16597 & 0.09961 & 0.14380 & 0.18769 \\
Supplier 3 & 0.43261 & 0.49307 & 0.52588 & 0.45847 & 0.35149 \\
Supplier 4 & 0.23874 & 0.19707 & 0.24941 & 0.24320 & 0.27215 \\
\hline
\end{tabular}

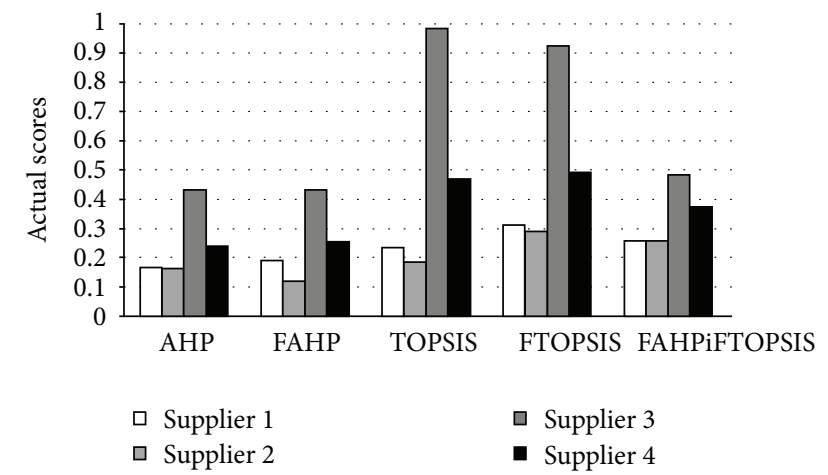

FIGURE 5: Summary of various decision making tools based on actual score.

tools applied. From this table, the values are then normalized as in Table 13. The equation used for normalization is presented in (34) below. The normalised scores in Table 13 were then used to plot the bar graph of Figure 6 . Normalized values are used to make values comparable with each other. It is a process of reducing the values into a standard scale [43].

Normalized value:

Actual score

Total actual score of the related decision making tool

Generally, it can be observed from Figure 6 that the ranking of supplier selection process for each different decision making tool followed the order of S3 $>$ S4 $>$ S2 $>$ S1 where Supplier 3 exhibits the highest priority level while Supplier 1 exhibits the lowest priority level. The normalized score for Supplier 3 hits above average of 0.3 , whereas for Supplier 1 and 2 the difference in normalized scoring is very small which indicates that both suppliers are competitively comparable.

The scores for the ranking among the four suppliers in each MCDM tool (AHP, FAHP, TOPSIS, and FTOPSIS) show significantly comparable variation. The percentage of variation scores for 4 suppliers is $-36.9 \%,-40.33 \%, 26.8 \%$, and $32.2 \%$ when comparing AHP, FAHP, TOPSIS, and

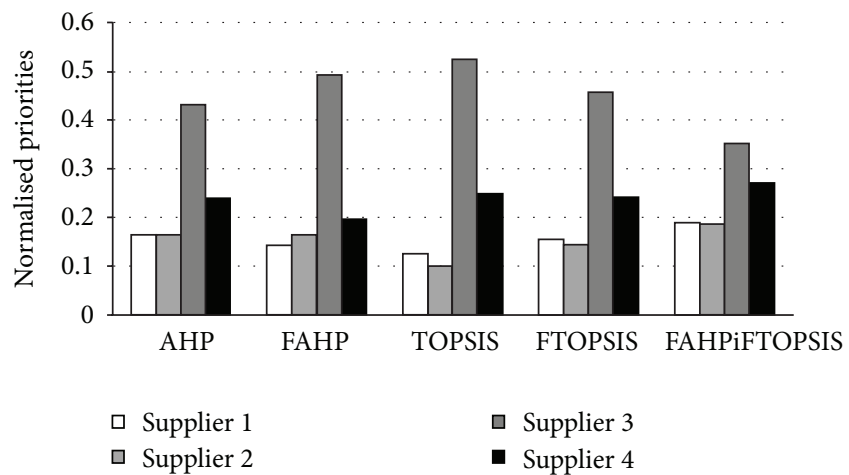

FIGURE 6: Summary of various decision making tools based on normalised priority.

FTOPSIS with FAHPiFTOPSIS, respectively. These variations are obtained from the average of the original scoring and then compared with respect to FAHPiFTOPSIS. Table 14 showed the variations of models with respect to each other. The computation can be performed as in (34) whereby the calculation for AHP percentage of variation is taken here, for example. The reliability of the model is based on the less variation of a model. Based on Table 14, when the variations are summed up for each MCDM model, FAHPiFTOPSIS achieved less variation, whereas the high variation model is FAHP for 3 computations with respect to FAHPiFTOPSIS, FTOPSIS, and TOPSIS

Percentage of variation:

$$
\begin{aligned}
& \text { ( } \\
& \text { average of AHP actual score-average of FAHPiFTOPSIS }
\end{aligned}
$$

The following evaluation to determine the variation is based on the difference of scores achieved by the best supplier with the less preferred supplier, and Table 15 is generated. The data for Table 15 can be obtained from Table 13. Referring to Table 15 and Figure 7, the highest percentage goes to 
TABLE 14: Variations of models with respect to each other.

\begin{tabular}{|c|c|c|c|c|c|}
\hline & AHP & FAHP & TOPSIS & FTOPSIS & FAHPiFTOPSIS \\
\hline With respect to AHP & - & $-2.5 \%$ & $46.5 \%$ & $50.5 \%$ & $26.9 \%$ \\
\hline With respect to FAHP & $2.4 \%$ & - & $47.8 \%$ & $51.7 \%$ & $28.7 \%$ \\
\hline With respect to TOPSIS & $-87 \%$ & $-91.7 \%$ & - & $7.4 \%$ & $-36.6 \%$ \\
\hline With respect to FTOPSIS & $-101.9 \%$ & $-107 \%$ & $-7.9 \%$ & - & $-47.5 \%$ \\
\hline With respect to FAHPiFTOPSIS & $-36.9 \%$ & $-40.33 \%$ & $26.8 \%$ & $32.2 \%$ & - \\
\hline Total percentage of variations & $-223.5 \%$ & $241.53 \%$ & $113.2 \%$ & $141.8 \%$ & $-28.5 \%$ \\
\hline
\end{tabular}

TABLE 15: Difference of normalized scores for the best supplier (Supplier 3) and the less preferred supplier (Supplier 2).

\begin{tabular}{|c|c|c|c|c|c|}
\hline & AHP & FAHP & TOPSIS & FTOPSIS & FAHPiFTOPSIS \\
\hline Supplier 2 & 0.16414 & 0.16597 & 0.09961 & 0.14380 & 0.18769 \\
\hline Supplier 3 & 0.43261 & 0.49307 & 0.52588 & 0.45847 & 0.35149 \\
\hline Score difference & 0.26847 & 0.29491 & 0.79734 & 0.63529 & 0.22422 \\
\hline Percentage & 26.84 & 29.49 & 79.73 & 63.53 & 22.42 \\
\hline
\end{tabular}

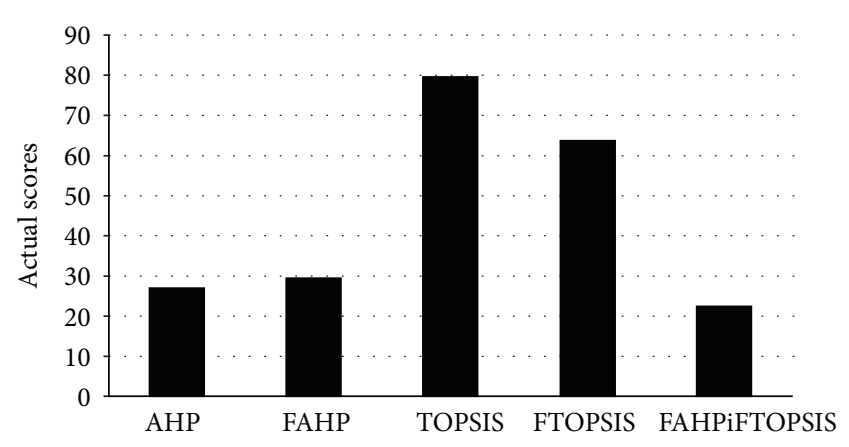

FIGURE 7: Difference in percentage comparison between the best supplier and the less preferred supplier.

TOPSIS with scoring of $79.73 \%$ followed by FTOPSIS with percentage of 63.53 and FAHP with $29.49 \%$. On the contrary, FAHPiFTOPSIS decision making tool demonstrated the lowest score variation of $22.42 \%$ followed by $26.84 \%$ of AHP. This indicates that FAHPiFTOPSIS is able to eliminate the "biasness" and ambiguity in supplier selection process.

\section{Conclusions}

This research has successfully demonstrated the applicability of the MCDM tools (AHP, FAHP, TOPSIS, FTOPSIS, and FAHPiFTOPSIS) by automotive industry in the selection of suppliers. As a result, few points can be concluded as follows.

(1) Six additional parameters were observed to have equal important criteria in automotive industry besides three classic criteria, namely, price, delivery, and quality which are support service, technology, factory capacity and capability, supplier background, flexibility, and other management system requirements.

(2) AHP, FAHP, TOPSIS, FTOPSIS, and FAHPiFTOPSIS are all applicable and accurate for supplier selection in automotive industry. (a) For AHP and FAHP, when the number of suppliers and alternatives becomes big, more criteria will need to be considered and decision maker will face a challenge to perform pairwise comparison in a big matrix.

(b) For TOPSIS and FTOPSIS, it is a simpler method whereby weights are needed as the input of this decision making tool irrespective with the number of alternatives.

(c) The results from AHP, FAHP, TOPSIS, and FTOPSIS show great variation in the final ranking scores. However, FAHPiFTOPSIS decision making tool demonstrated the lowest score variation of $22.42 \%$. This indicates that FAHPiFTOPSIS application is able to effectively eradicate any ambiguity or fuzziness in the process.

\section{Appendices}

\section{A. Supplier Selection Criteria}

See Table 16.

\section{B. Generic System Architecture of FAHP, FTOPSIS, and FAHPiFTOPSIS for Decision Makers in Selecting the Best Supplier}

See Figure 8.

\section{Questionnaire Developed for the Study}

C.1. Developed Questionnaire. The developed questionnaire is called:

$\underline{\text { Supplier selection process for automotive industry. }}$

C.1.1. Supplier Selection Criteria. Supplier selection evaluations are normally being conducted by companies in 
TABLE 16: Supplier selection criteria.

\begin{tabular}{ll}
\hline Category & Criteria \\
\hline & (i) Applies JIT concept \\
(1) Delivery & (ii) Delivery lead time \\
& (iii) Delivery quality \\
& (iv) Packaging quality \\
& (i) Handled by expertise of the related field \\
(2) Support & (ii) Availability \\
service & (iii) Promptness \\
& (iv) Applies OEE concept \\
& (i) Quality performance \\
(3) Quality & (ii) Durability \\
& (iii) Ergonomic qualities \\
& (iv) Reliability
\end{tabular}

(i) Collaboration with established R\&D organization on referral designs

(ii) Product certification

(iii) High technology machines and processes

(iv) Align with current technology (product/process/design)

(v) Suppliers capable of modifying product/design/process

(vi) Material availability

(vii) Alternative material and technologies

(4) Technology

(viii) EDI capability
(a) inventory
(b) logistics
(c) production
(d) transaction

(ix) Design capability
(a) Flexibility to respond to design changes
(b) Product innovativeness
(c) Product performance
(d) Ability to modify product/process

(x) R\&D
(a) Availability of testing laboratory
(b) Reliability of testing laboratory
(c) Calibration program

(i) Flexibility in price reduction

(5) Price

(ii) Competitive operating costs

(iii) Flexibility in payment

(6) Factory capacity and capability

(i) Level of capability to cope with rush orders

(ii) Sufficient product capacity

(iii) Sufficient product facilities
TABLE 16: Continued.

\begin{tabular}{l} 
Category \\
\hline Criteria \\
(i) Industry knowledge (specifically on the \\
related process or parts) \\
(ii) Geographical location of the supplier \\
(iii) Geographical condition such as labor \\
cost and traffic congestion \\
(a) suppliers have dedicated supply \\
point
\end{tabular}

(7) Supplier background

(iv) Flexibility in freight
(a) suppliers have their own transportation

(v) Position in the industry and reputation
(a) suppliers supply to other established car manufacturers

(vi) Performance history
(a) suppliers have been blacklisted or not

(vii) Financial
(a) Table financial management system
(b) bankruptcy

(viii) Manning
(a) sufficient workers
(b) low turnover rate

(i) Practices flexible manufacturing system in terms of design and process

(8) Flexibility

(ii) Flexibility of operation

(iii) Flexibility in production

(iv) Flexibility in order frequency and amount

(v) Flexibility to respond requirement volume changes

\begin{tabular}{ll}
\hline & (i) Kanban \\
& (ii) Kaizen \\
& (iii) TPS (Toyota production system) \\
& (iv) ISO 9000 \\
(9) Management & (v) Six sigma \\
tool system & (vi) 5 S \\
& (vii) FMEA \\
& (viii) SPC \\
& (ix) Strong safety processes and culture \\
& (x) Environmental performance \\
\hline
\end{tabular}

order to evaluate their suppliers' performance and reliability. Traditionally, supplier selection evaluations are based on price/cost, quality, and delivery. However, in today's management, most companies do not only consider the three primary criteria, but they also take into account technology, support 
TABLE 17

\begin{tabular}{l}
\hline Criteria \\
\hline (1) On time delivery (minimize lateness and earliness) \\
(2) Proper delivery record and followup \\
(3) Minimum shipping error (number of incorrect shipment) \\
(4) Able to deliver supplies within short lead time \\
(5) Products are checked thoroughly prior delivery \\
(6) Applies JIT (just in time) concept
\end{tabular}

TABLE 18

Criteria

(1) Provide supporting staff to overcome any technical and supply problems

(2) Supporting staff and technical experts are readily available

(3) Promptness to response

(4) Professional attitude in dealing with problems

TABLE 19

Criteria

(1) Meeting customer requirement and expectations

(2) Zero reject rate

(3) Reliability in raw material supplies

(4) Conformance to environmental standard specifications

(5) Proper record on complaints and followup

(6) Provide sample of components or raw material before delivery

(7) Ergonomic quality in product/raw material design

(8) Special packaging and shipping requirement

(9) Proper marking and labeling of materials (legible, durable, and follow specification)

(10) Safety record of suppliers

TABLE 20

Criteria

(1) Technological capabilities for future improvement

(2) Collaboration with established R\&D organization or institution

(3) Information system capabilities (electronic data interchanges, ERP, barcode)

(4) Innovativeness in product development and improvement

(5) Have intellectual property (trademarks, patents) and unique technologies capability

(6) Product development facility

(7) Efficient production facilities and capacity

(8) Complying to international certifications

service, flexibility, supplier background, management tool systems, and factory capacity and capability.

As part of my Master research in manufacturing engineering, I would like to seek you a great assistance to provide me your critical input on supplier selection. Below you will
TABLE 21

Criteria

(1) Low or reasonable price of supplies

(2) Minimize distribution/logistic costs (transportation, administrative, customs, risk, and handling)

(3) Avoid additional cost incurred in manufacturing, labor, maintenance, inspection, rework, or due to raw materials

(4) Eliminate after sales service costs

(5) Additional discount for large volume purchase

(6) Additional cost required due to low volume ordered

TABLE 22

Criteria

(1) Manufacturing capability to cope with rush orders

(2) Sufficient product capacity

(3) Sufficient product facilities

TABLE 23

Criteria

(1) Experts in the industry

(2) Suppliers having own transportation

(3) Suppliers having strong financial standing

(4) Suppliers having sufficient workers

(5) Strategic geographical location

TABLE 24

Criteria

(1) Flexible in design and process

(2) Flexibility in manufacturing operations

(3) Flexibility in production planning

(4) Flexibility in order frequency and amount

(5) Flexibility in response to changes on requirement

(6) Speedy product conception to product realization

find the criteria listed. What you have to do is to fill in your inputs in each question by writing the numbers according to the ranking in the right box.

(1) Delivery/Lead Time. Delivery of supply: whichever is applied to your company, please indicate the degree of importance on the following delivery criteria as expected from your suppliers. See Table 17.

(2) Support Service. See Table 18.

(3) Quality Factor. Supplier selection is not only limited to cost, but also to the performance of other competitive dimensions of quality. Meeting customer requirements is one example, but there are other important factors involved. Please indicate the degree of importance on the following quality criteria as expected from your suppliers. See Table 19. 
TABLE 25

Criteria

(1) Kanban

(2) Kaizen

(3) TPS (Toyota production system)

(4) ISO 9000

(5) Six sigma

(6) $5 \mathrm{~S}$

(7) FMEA

(8) SPC

(9) TS16949

(10) Safety management and culture

(11) Takt time

(12) Overall equipment effectiveness

TABLE 26

Criteria Supplier 1 Supplier 2 Supplier 3 Supplier 4

(1) Delivery/lead time

(2) Support service

(3) Quality factor

(4) Technology

(5) Price/cost

(6) Factory capacity

and capability

(7) Supplier background

(8) Flexibility

(9) Management system

Criteria rating: (1) Very poor $\rightarrow$ (9) very good.

TABLE 27: Saaty's pair-wise comparison table.

\begin{tabular}{lc}
\hline Numerical rating & Verbal comparison of preference \\
\hline 1 & Equal importance \\
3 & Moderate importance of one over another \\
5 & Strong or essential importance \\
7 & Very strong or demonstrated importance \\
9 & Extreme importance \\
$2,4,6,8$ & Intermediate values \\
\hline
\end{tabular}

(Source: [29]).

(4) Technology. Suppliers have to cope with technology to be more competitive. Suppliers which are responsive to new technology are more favorable to any company. Please

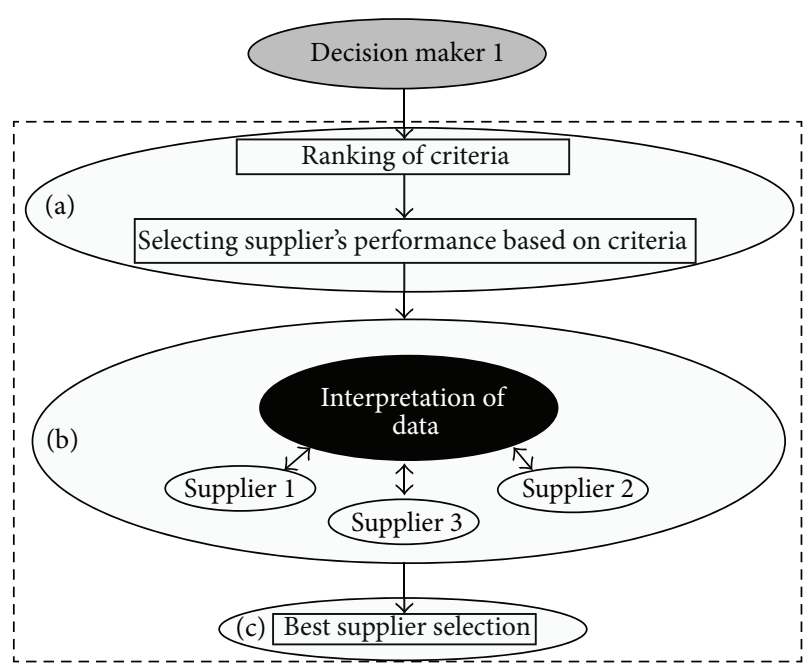

Figure 8

indicate the degree of importance on the following criteria. See Table 20.

(5) Price/Cost. Traditionally, any company will go for the lowest cost from their suppliers. Consequently, the cost control system administered by any company may have some effect on the suppliers. The criteria below show some of the important issues related to cost and price. Please indicate their level of importance as applicable to your company. See Table 21.

(6) Factory Capacity and Capability. See Table 22.

(7) Supplier Background. The additional requirements criteria involve supplier's background. Below are some of the important factors for suppliers. Please indicate their level of importance. See Table 23.

(8) Flexibility. See Table 24.

(9) Management System. See Table 25.

C.1.2. Rating of Suppliers under Various Criteria. Please state your level of importance of the supplier with respect to the criteria on the right boxes regarding the numbers below. See Table 26.

C.1.3. Pairwise Comparison of 9 Criteria. According to Saaty's table, compare the 9 criteria accordingly and fill in the table. Criteria on the left side of the table need to be compared to the criteria at the horizontal columns. See Tables 27 and 28.

C.1.4. Pairwise Comparison of Suppliers with Respect to 9 Criteria. According to Saaty's table, compare the suppliers (S1, S2, S3, and S4) with respect to the criteria. Suppliers on the left side columns are to be compared with the suppliers on the right columns. Kindly mark (/) at the preferred value when pairwise comparison is performed. See Tables 29, 30, $31,32,33,34,35,36,37$, and 38. 
TABle 28

\begin{tabular}{|c|c|c|c|c|c|c|c|c|c|}
\hline \multirow{2}{*}{ Criteria 1} & Criteria 1 & Criteria 2 & Criteria 3 & Criteria 4 & Criteria 5 & Criteria 6 & Criteria 7 & Criteria 8 & Criteria 9 \\
\hline & & & & & & & & & \\
\hline Criteria 2 & & & & & & & & & \\
\hline Criteria 3 & & & & & & & & & \\
\hline Criteria 4 & & & & & & & & & \\
\hline Criteria 5 & & & & & & & & & \\
\hline Criteria 6 & & & & & & & & & \\
\hline Criteria 7 & & & & & & & & & \\
\hline Criteria 8 & & & & & & & & & \\
\hline Criteria 9 & & & & & & & & & \\
\hline
\end{tabular}

TABLE 29: Saaty's pair-wise comparison table.

\begin{tabular}{lc}
\hline Numerical rating & Verbal comparison of preference \\
\hline 1 & Equal importance \\
3 & Moderate importance of one over another \\
5 & Strong or essential importance \\
7 & Very strong or demonstrated importance \\
9 & Extreme importance \\
$2,4,6,8$ & Intermediate values \\
\hline
\end{tabular}
(Source: [29]).

TABle 30: Criterion 1: delivery/lead time.

\begin{tabular}{llllllllllllllllll}
\hline & 9 & 8 & 7 & 6 & 5 & 4 & 3 & 2 & 1 & 2 & 3 & 4 & 5 & 6 & 7 & 8 & 9 \\
\hline S1 & & & & & & & & & & & & & & & & & S2 \\
S1 & & & & & & & & & & & & & & & & & \\
S1 & & & & & & & & & & & & & & & & & S3 \\
S2 & & & & & & & & & & & & & & & & & S4 \\
S2 & & & & & & & & & & & & & & & & & S3 \\
S3 & & & & & & & & & & & & & & & & & \\
\hline
\end{tabular}

TABLE 31: Criterion 2: support service.

\begin{tabular}{|c|c|c|c|c|c|c|c|c|c|c|c|c|c|c|c|c|}
\hline 9 & 8 & 7 & 6 & 5 & 4 & 3 & 2 & 1 & 2 & 3 & 4 & 5 & 6 & 7 & 8 & 9 \\
\hline S1 & & & & & & & & & & & & & & & & S2 \\
\hline S1 & & & & & & & & & & & & & & & & S3 \\
\hline S1 & & & & & & & & & & & & & & & & S4 \\
\hline S2 & & & & & & & & & & & & & & & & S3 \\
\hline S2 & & & & & & & & & & & & & & & & S4 \\
\hline S3 & & & & & & & & & & & & & & & & S4 \\
\hline
\end{tabular}

TABLE 32: Criterion 3: quality.

\begin{tabular}{llllllllllllllllll}
\hline & 9 & 8 & 7 & 6 & 5 & 4 & 3 & 2 & 1 & 2 & 3 & 4 & 5 & 6 & 7 & 8 & 9 \\
\hline S1 & & & & & & & & & & & & & & & & \\
S1 & & & & & & & & & & & & & & & & \\
S1 & & & & & & & & & & & & & & & & & \\
S2 & & & & & & & & & & & & & & & & \\
S2 & & & & & & & & & & & & & & & & & S4 \\
S3 & & & & & & & & & & & & & & & & S3 \\
\hline
\end{tabular}

TABLE 33: Criterion 4: technology.

\begin{tabular}{llllllllllllllllll}
\hline & 9 & 8 & 7 & 6 & 5 & 4 & 3 & 2 & 1 & 2 & 3 & 4 & 5 & 6 & 7 & 8 & 9 \\
\hline S1 & & & & & & & & & & & & & & & & \\
S1 & & & & & & & & & & & & & & & & \\
S1 & & & & & & & & & & & & & & & & S3 \\
S2 & & & & & & & & & & & & & & & & S4 \\
S2 & & & & & & & & & & & & & & & & S3 \\
S3 & & & & & & & & & & & & & & & & \\
\hline
\end{tabular}

TABLE 34: Criterion 5: price/cost.

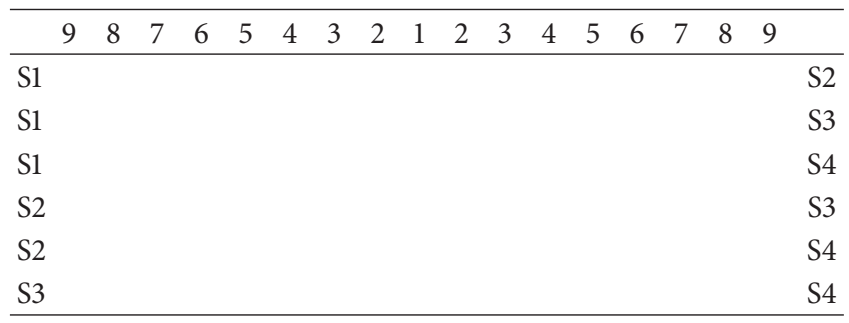

TABLE 35: Criterion 6: factory capacity and capability.

\begin{tabular}{llllllllllllllllll}
\hline & 9 & 8 & 7 & 6 & 5 & 4 & 3 & 2 & 1 & 2 & 3 & 4 & 5 & 6 & 7 & 8 & 9 \\
\hline S1 & & & & & & & & & & & & & & & & & S2 \\
S1 & & & & & & & & & & & & & & & & \\
S1 & & & & & & & & & & & & & & & & S3 \\
S2 & & & & & & & & & & & & & & & & S4 \\
S2 & & & & & & & & & & & & & & & & & S3 \\
S3 & & & & & & & & & & & & & & & & S4 \\
\hline
\end{tabular}

TABLE 36: Criterion 7: supplier background.

\begin{tabular}{lrrrrrrrrrrrrrrrrr}
\hline & 9 & 8 & 7 & 6 & 5 & 4 & 3 & 2 & 1 & 2 & 3 & 4 & 5 & 6 & 7 & 8 & 9 \\
\hline S1 & & & & & & & & & & & & & & & & \\
S1 & & & & & & & & & & & & & & & & & \\
S1 & & & & & & & & & & & & & & & & & S3 \\
S2 & & & & & & & & & & & & & & & & & S4 \\
S2 & & & & & & & & & & & & & & & & & S3 \\
S3 & & & & & & & & & & & & & & & & & \\
\hline
\end{tabular}


TABLE 37: Criterion 8: flexibility.

\begin{tabular}{llllllllllllllllll}
\hline & 9 & 8 & 7 & 6 & 5 & 4 & 3 & 2 & 1 & 2 & 3 & 4 & 5 & 6 & 7 & 8 & 9 \\
\hline S1 & & & & & & & & & & & & & & & & & \\
S1 & & & & & & & & & & & & & & & & & \\
S1 & & & & & & & & & & & & & & & & & S3 \\
S2 & & & & & & & & & & & & & & & & & S4 \\
S2 & & & & & & & & & & & & & & & & & S3 \\
S3 & & & & & & & & & & & & & & & & & S4 \\
\hline
\end{tabular}

TABLE 38: Criterion 9: management tool system.

\begin{tabular}{lrrrrrrrrrrrrrrrrr}
\hline & 9 & 8 & 7 & 6 & 5 & 4 & 3 & 2 & 1 & 2 & 3 & 4 & 5 & 6 & 7 & 8 & 9 \\
\hline S1 & & & & & & & & & & & & & & & & & S2 \\
S1 & & & & & & & & & & & & & & & & & \\
S1 & & & & & & & & & & & & & & & & & S3 \\
S2 & & & & & & & & & & & & & & & & & S4 \\
S2 & & & & & & & & & & & & & & & & & S3 \\
S3 & & & & & & & & & & & & & & & & & \\
\hline
\end{tabular}

\section{Acknowledgments}

The authors would like to thank Associate Professor Dr. Mohd. Khaled Omar and all the survey respondents for their kind support and help contributed towards the implementation of this paper.

\section{References}

[1] V. Vaidya, Impact of Thailand Floods on Automotive Industry and Supply Chain, Frost and Sullivan, 2011.

[2] C. T. Chen, C. T. Lin, and S. F. Huang, "A fuzzy approach for supplier evaluation and selection in supply chain management," International Journal of Production Economics, vol. 102, no. 2, pp. 289-301, 2006.

[3] Malaysia Automotive Association, MAA, Summary of Sales \& Production Data, 2011.

[4] A. Ahmad, Market Review of 2010 and Outlook for 2011, Malaysia Automotive Association, 2011.

[5] Secretariat, ASEAN Free Trade Area (AFTA) : An update, Association of Southeast Nation, 1999.

[6] Bernama, "DRB-Hicom's Pekan Plant to See Higher Production," Bernama, 2009.

[7] C. Kahraman, U. Cebeci, and Z. Ulukan, "Multi-criteria supplier selection using fuzzy AHP," Logistics Information Management, vol. 16, pp. 382-394, 2003.

[8] T. L. Saaty, The Analytical Hierarchy Process, McGrawHill, New York, NY, USA, 1980.

[9] T. L. Saaty, "Axiomatic foundation of the analytical hierarchy process," Management Science, vol. 32, no. 7, pp. 841-855, 1986.

[10] O. Cakir and M. S. Canbolat, "A web-based decision support system for multi-criteria inventory classification using fuzzy AHP methodology," Expert Systems with Applications, vol. 35, no. 3, pp. 1367-1378, 2008.

[11] C. L. Hwang and K. Yoon, Multiple Attribute Decision MakingMethods and Applications, Springer, Heidelberg, Germany, 1981.

[12] L. De Boer, L. Van Der Wegen, and J. Telgen, "Outranking methods in support of supplier selection," European Journal of
Purchasing and Supply Management, vol. 4, no. 2-3, pp. 109-118, 1998.

[13] J. Wang, K. Fan, and W. Wang, "Integration of fuzzy AHP and FPP with TOPSIS methodology for aeroengine health assessment," Expert Systems with Applications, vol. 37, no. 12, pp. 8516-8526, 2010.

[14] L. A. Zadeh, "Fuzzy sets," Information and Control, vol. 8, no. 3, pp. 338-353, 1965.

[15] M. Negnevitsky, Artificial Intelligence: A Guide To Intelligent Systems, Addison-Wesley: Pearson Education Limited, Essex, UK, 2002.

[16] M. Bevilacquaa, F. E Ciarapicab, and G. Giacchettab, "A fuzzyQFD approach to supplier selection," Journal of Purchasing \& Supply Management, vol. 12, pp. 14-27, 2006.

[17] H. Deng, "Multicriteria analysis with fuzzy pair-wise comparison," International Journal of Approximate Reasoning, vol. 21, pp. 215-231, 1999.

[18] S. M. Ordoobadi, "Development of a supplier selection model using fuzzy logic," Supply Chain Management, vol. 14, no. 4, pp. 314-327, 2009.

[19] T. L. Saaty, "A scaling method for priorities in hierarchical structures," Journal of Mathematical Psychology, vol. 15, no. 3, pp. 234-281, 1977.

[20] C. C. Yang and B. S. Chen, "Supplier selection using combined analytical hierarchy process and grey relational analysis," Journal of Manufacturing Technology Management, vol. 17, no. 7, pp. 926-941, 2006.

[21] R. L. Nydick and R. P. Hill, "Using the analytic hierarchy process to structure the supplier selection procedure," International Journal of Purchasing and Materials Management, pp. 31-36, 1992.

[22] I. Ertuğrul and N. Karakaşoğlu, "Comparison of fuzzy AHP and fuzzy TOPSIS methods for facility location selection," International Journal of Advance Manufacturing Technology, vol. 39, pp. 783-795, 2008.

[23] F. Tüysüz and C. Kahraman, "Project risk evaluation using a fuzzy analytic hierarchy process: an application to information technology projects," International Journal of intelligent Systems, vol. 21, pp. 559-584, 2006.

[24] A. Kelemenis, K. Ergazakis, and D. Askounis, "Support managers' selection using an extension of fuzzy TOPSIS," Expert Systems with Applications, vol. 38, no. 3, pp. 2774-2782, 2011.

[25] E. Bottani and A. Rizzi, "A fuzzy TOPSIS methodology to support outsourcing of logistics services," Supply Chain Management, vol. 11, no. 4, pp. 294-308, 2006.

[26] H. J. Shyur, "COTS evaluation using modified TOPSIS and ANP," Applied Mathematics and Computation, vol. 177, no. 1, pp. 251-259, 2006.

[27] M. F. Shipley, A. de Korvin, and R. Obid, "A decision making model for multi-attribute problems incorporating uncertainty and bias measures," Computers and Operations Research, vol. 18, no. 4, pp. 335-342, 1991.

[28] O. Durán and J. Aguilo, "Computer-aided machine-tool selection based on a Fuzzy-AHP approach," Expert Systems with Applications, vol. 34, no. 3, pp. 1787-1794, 2008.

[29] T. L. Saaty, Multi-Criteria Decision Making: the Analytic Hierarchy Process-Planning, Priority Setting, Resource Allocation, R W S Publications, Pittsburgh, Pa, USA, 1996.

[30] C. C. Sun, "A performance evaluation model by integrating fuzzy AHP and fuzzy TOPSIS methods," Expert Systems with Applications, vol. 37, no. 12, pp. 7745-7754, 2010. 
[31] P. J. M. van Laarhoven and W. Pedrycz, "A fuzzy extension of Saaty's priority theory," Fuzzy Sets and Systems, vol. 11, no. 3, pp. 229-241, 1983.

[32] D. Y. Chang, Extent Analysis and Synthetic Decision, Optimization Techniques and Applications, vol. 1, World Scientific, Singapore, 1992.

[33] A. T. Gumus, "Evaluation of hazardous waste transportation firms by using a two step fuzzy-AHP and TOPSIS methodology," Expert Systems with Applications, vol. 36, no. 2, pp. 40674074, 2009.

[34] D. L. Olson, "Comparison of weights in TOPSIS models," Mathematical and Computer Modelling, vol. 40, no. 7-8, pp. 721727, 2004.

[35] R. A. Krohling and V. C. Campanharo, "Fuzzy TOPSIS for group decision making: a case study for accidents with oil spill in the sea," Expert Systems with Applications, vol. 38, no. 4, pp. 41904197, 2011.

[36] C. T. Chen, "Extensions of the TOPSIS for group decisionmaking under fuzzy environment," Fuzzy Sets and Systems, vol. 114, no. 1, pp. 1-9, 2000.

[37] T. Y. Chen and C. Y. Tsao, “The interval-valued fuzzy TOPSIS method and experimental analysis," Fuzzy Sets and Systems, vol. 159, no. 11, pp. 1410-1428, 2008.

[38] Y. M. Wang and T. M. S. Elhag, "Fuzzy TOPSIS method based on alpha level sets with an application to bridge risk assessment," Expert Systems with Applications, vol. 31, no. 2, pp. 309-319, 2006.

[39] D. Yong, "Plant location selection based on fuzzy TOPSIS," International Journal of Advanced Manufacturing Technology, vol. 28, no. 7-8, pp. 839-844, 2006.

[40] J.-J. Buckley, "Fuzzy hierarchical analysis," Fuzzy Sets and Systems, vol. 17, no. 1, pp. 233-247, 1985.

[41] U. Nirmal, B. F. Yousif, D. Rilling, P. V. Brevern, and N. Jamil, "The potential of using treated betelnut fibres as reinforcement for tribo-bio polymeric composites subjected to dry/wet contact conditions," in Proceedings of the International Conference on Natural Polymers (ICNP '10), Kottayam, Kerala, India, September 2010.

[42] U. Nirmal, N. Singh, J. Hashim, S. T. W. Lau, and N. Jamil, "On the effect of different polymer matrix and fibre treatment on single fibre pullout test using betelnut fibres," Materials and Design, vol. 32, no. 5, pp. 2717-2726, 2011.

[43] S. Borgatti, Research Method, BA 762, May 2012. 

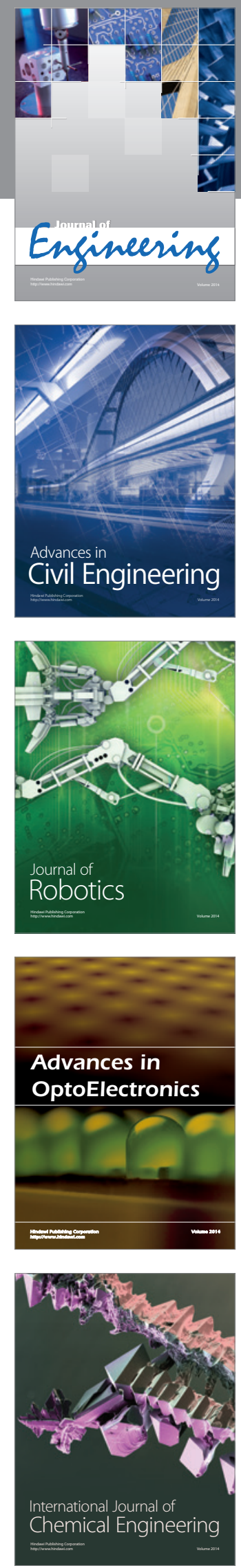

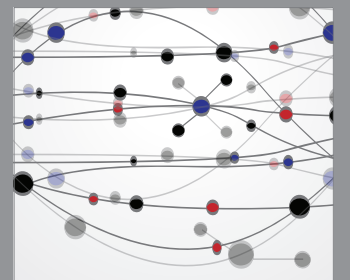

The Scientific World Journal
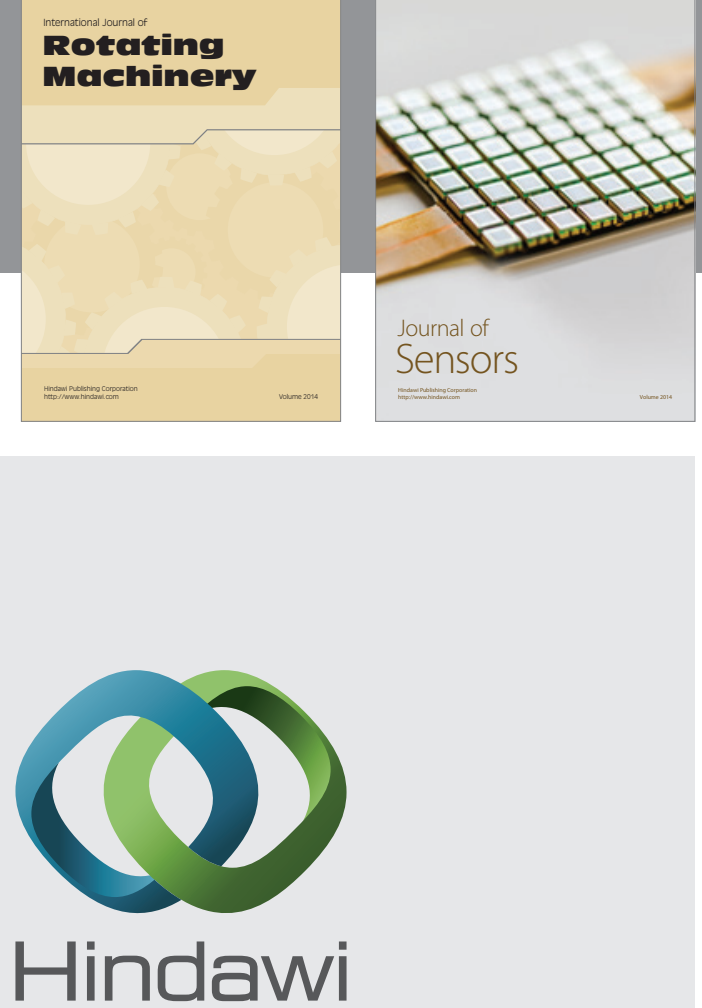

Submit your manuscripts at http://www.hindawi.com
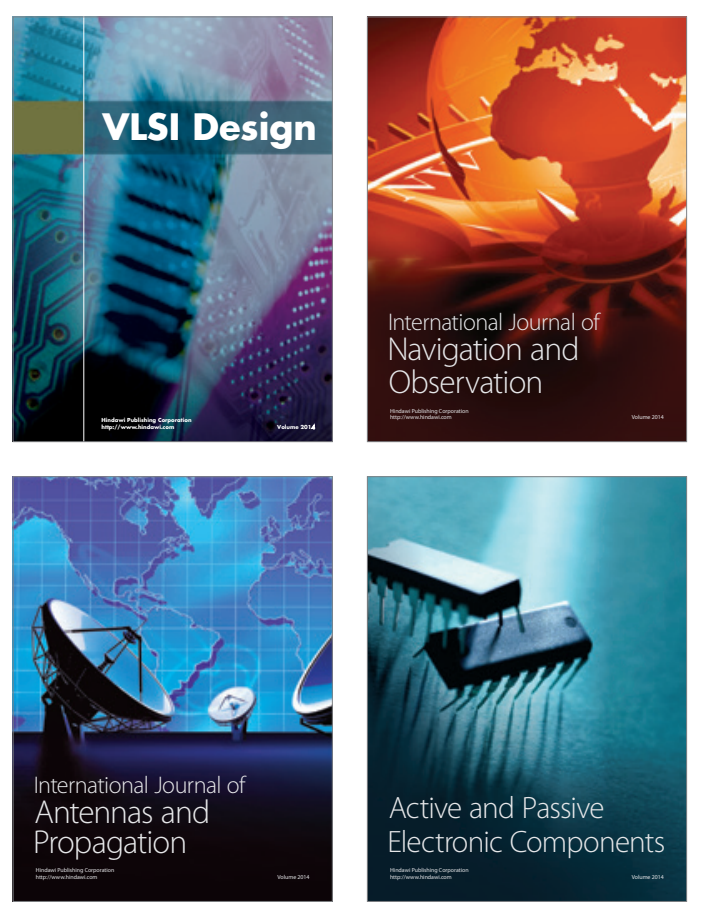
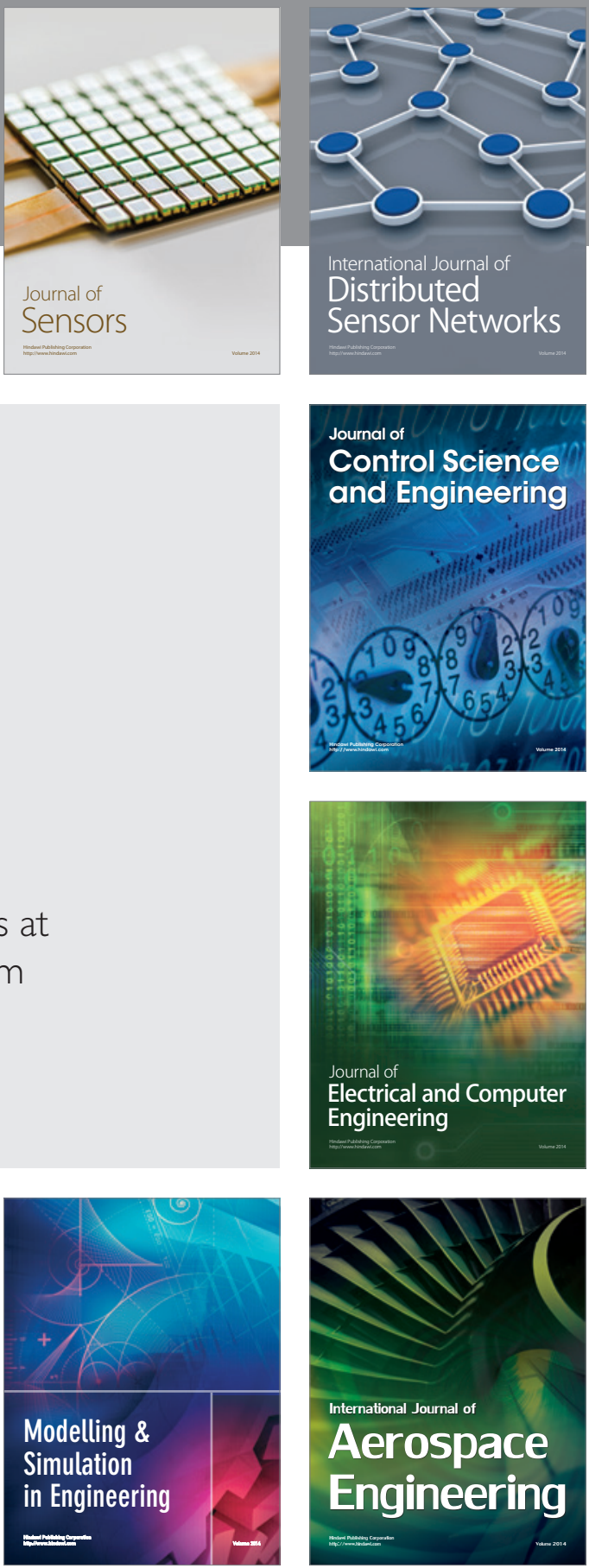

Journal of

Control Science

and Engineering
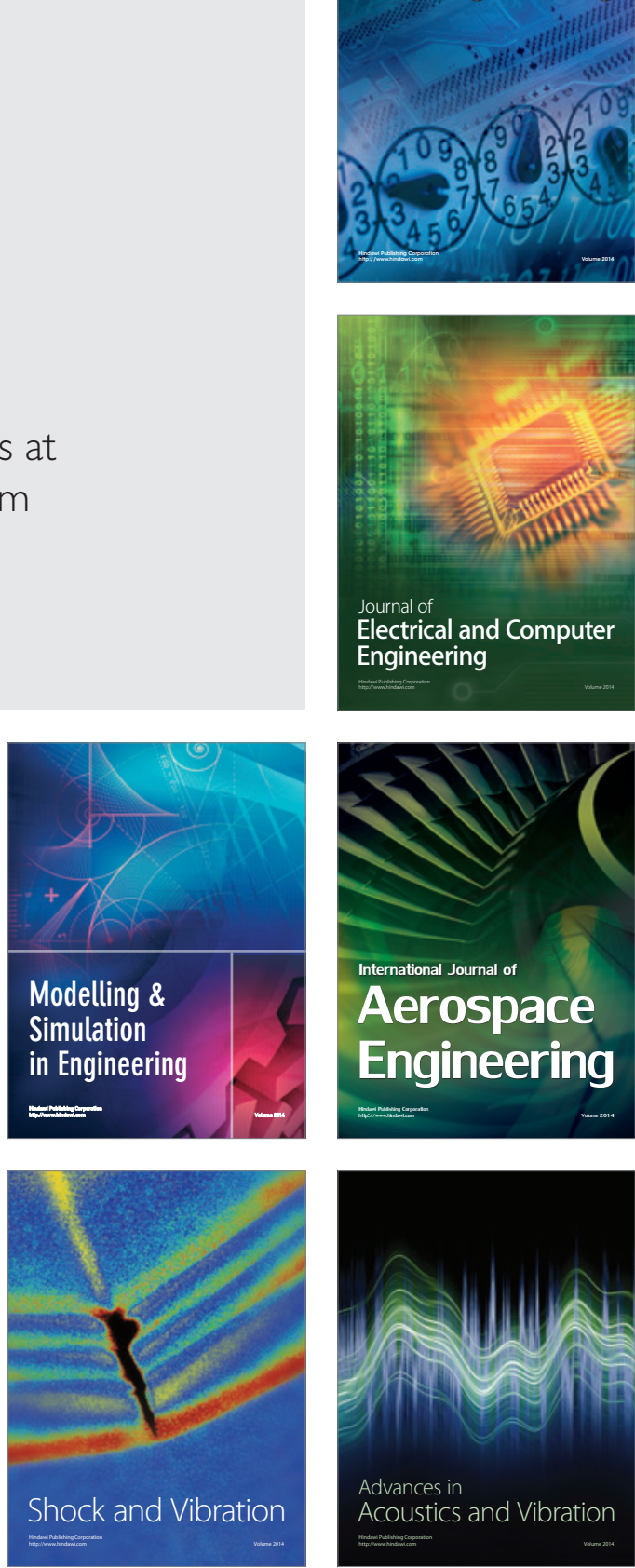